CERN-TH. 7206/94

\title{
ANALYTIC SOLUTIONS OF QCD EVOLUTION EQUATIONS FOR PARTON CASCADES INSIDE NUCLEAR MATTER AT SMALL X
}

\author{
K. Geiger \\ CERN TH-Division, CH-1211 Geneva 23, Switzerland
}

\begin{abstract}
An analytical method is presented to solve generalized QCD evolution equations for the time development of parton cascades in a nuclear environment. In addition to the usual parton branching processes in vacuum, these evolution equations provide a consistent description of interactions with the nuclear medium by accounting for stimulated branching processes, fusion and scattering processes that are specific to QCD in medium. Closed solutions for the spectra of produced partons with respect to the variables time, longitudinal momentum and virtuality are obtained under some idealizing assumptions about the composition of the nuclear medium. Several characteristic features of the resulting parton distributions are discussed. One of the main conclusions is that the evolution of a parton shower in medium is dilated as compared to free space and is accompanied by an enhancement of particle production. These effects become stronger with increasing nuclear density.
\end{abstract}

PACS Indices: 25.75.+r, 12.38.Bx, 12.38.Mh, 24.85.+p

e-mail: klaus@surya11.cern.ch

CERN-TH. 7206/94, March 1994 


\section{INTRODUCTION}

In a preceding paper [1], B. Müller and myself addressed the question, how is "QCD in medium" modified as compared to "QCD in vacuum" when one attempts to extend well developed perturbative QCD techniques [2] from high energy hadron-hadron collisions to ultra-relativistic heavy ion collisions. The investigation of [1] ] condensed in the derivation of a generalized form of QCD evolution equations that describe the time evolution of parton distributions in a nuclear medium, solutions of which I will present in this paper. This aspect is of great importance for the future ultra-relativistic heavy ion collider experiments at the BNL Relativistic Heavy Ion Collider (RHIC) and the CERN Large Hadron Collider (LHC), where new phenomena associated with "QCD in medium" are expected to clearly modify naive extrapolations from hadron-hadron collisions. Although in the last couple of years, numerical simulations with QCD based Monte Carlo models [3 6] have provided considerable new insight in the microscopic parton dynamics of ultra-relativistic heavy ion collisions, one is still far from a complete picture and a truly quantitative description. Aside from the rather intransparent complexity of these computer simulations, one still relies on a substantial amount of phenomenological modeling of nuclear and dense medium effects due the current lack of better knowledge about the details of such mechanisms. This is reflected by a very large uncertainty in quantitative predictions: although the different model calculations generally agree very well for $p p$ collisions at collider energies, they differ in their predictions, for e.g. charged particle multiplicities, in heavy ion $A A$ collisions by a factor of 2 or more.

These issues lead to the main motivation for this work, namely the necessity to go back to a simpler, but more fundamental and transparent level, and study within the firmly established framework of perturbative QCD (rather than extending phenomenological model input) the microscopic dynamics of quarks and gluons in the hot, ultra-dense environment that may be created in heavy ion collisions [7 [9]. In order to do so, it is inevitable to disentangle the various nuclear and medium effects from each other by investigating physical 
situations where such medium phenomena may be singled out, both in theoretical and experimental research. For instance, deep inelastic scattering on heavy nuclei [10], charmonium production by Drell-Yan processes in nuclei [11], energy loss of partons, jet quenching and multiple scattering phenomena in hadron-nucleus collisions [12], etc., provide opportunities to explore nuclear modifications to the QCD parton picture that has been so successful in high energy particle physics.

As a first step in this direction, in Ref. [1] a generalized form of the well known DokshitzerGribov-Lipatov-Altarelli-Parisi (DGLAP) evolution equations [13 15] was derived. In the framework of the leading logarithmic approximation (LLA) 16 19, we obtained a set of coupled integro-differential equations for the time evolution (rather than the $Q^{2}$-evolution) of the off-shell parton distribution functions in nuclear matter. In addition to the usual parton branching processes in vacuum, these evolution equations provide a self-contained description of interactions with the nuclear medium by accounting for stimulated branching processes, fusion and scattering processes, which are specific to QCD in medium. This approach resulted in a probabilistic description within perturbative QCD of the time variation of parton distributions as in non-equilibrium kinetic theory, however now including consistent treatment of off-shell propagation of partons, a concept that is absent in semiclassical transport theory [20]. The essential condition for the applicability of perturbative techniques is that the QCD factorization theorem [21] holds also in the presence of dense nuclear matter, in which multiple interactions of the evolving partons with this nuclear medium may be separated in space-time by only very short distances. Recent investigations by McLerran and Venugopalan [22] are encouraging in this direction, since they showed that a consistent perturbative calculation of parton structure functions at small values of the Bjorken variable $x$ becomes possible when one considers the limit of a very thick nuclear target. The condition for the applicability of perturbative QCD is then that medium induced effects, such as color screening and rescattering, provide dynamical cut-offs on a scale short compared to the QCD renormalization scale $\Lambda^{-1}$.

Exploring this insight, the present paper as a sequel to the work of Ref. [1] employs 
the probabilistic parton cascade picture [15,23] to describe the evolution of a parton shower inside nuclear matter, triggered by a highly excited initial quark or gluon. Here I will start from the generalized evolution equations and solve for the parton spectra with respect to the variables time, longitudinal momentum and virtuality, under some idealizing assumptions about the composition of the nuclear medium. Before going to the heart of the matter, let me remark the following: The approach is at this point in a yet idealized stage, since various unsettled problems are left out, and a number of simplifying approximations are made. Still, it is a starting point which can be step by step improved, similarly maybe as the development of perturbative QCD in hard processes that started some 15 years ago. The main assumptions and approximations in Ref. [1] and in the present paper can be stated as follows:

(i) Factorization of short distance interactions of shower partons with the nuclear medium from long range non-perturbative forces is assumed. This means, even in the presence of dense medium where a parton can encounter multiple successive interactions, a probabilistic description of local, non-interfering interactions applies at sufficiently high energies.

(ii) Neglect of interference effects that affect particularly the softest partons: destructive interference of interaction amplitudes in both, coherent succesive small angle emissions ('angular ordering'), and multiple sequential scatterings ('Landau-PomerantchukMigdal effect').

(iii) Nuclear effects due to long range correlations, e.g. nuclear shadowing (antishadowing) not associated with truly perturbative parton interactions are ignored.

The remainder of the paper is organized as follows. Sec. 2 is devoted to recall the intuitive picture developed in Ref. [1] of the probabilistic parton evolution in medium. The theoretical framework is reviewed and the master equations describing the time development of the distribution of off-shell partons in a nuclear environment are summarized. Sec. 3 then deals 
with linearizing and solving these equations analytically by making a number of simplifying assumptions regarding the nuclear medium. Explicit closed solutions for parton showers initiated by an energetic time-like quark or gluon are obtained, which in the special case of absence of nuclear matter reduces to the well known LLA solutions for parton distributions, however now time being the evolution variable rather than virtuality. Some general features concerning the $x$-dependence as well as the influence of the nuclear density are discussed. A summary and outlook is given in Sec. 4.

\section{THE COUPLED EVOLUTION EQUATIONS FOR QUARKS, ANTI- QUARKS AND GLUONS IN MEDIUM}

Let me start by recalling the essential considerations of Ref. [1] that lead to the derivation of a coupled set of integro-differential equations for a system of off-shell quarks (antiquarks) $q_{i}\left(\bar{q}_{i}\right)$ with flavors $i=1, \ldots, n_{f}$, and gluons $g$. As will become clear, these Boltzmann type equations describe the time evolution of parton cascades in a nuclear medium with account of off-shell propagation of time-like virtual partons. Although as mentioned before, the evolution equations may be applied to a variety of physical situations, let me specify here for the purpose of lucidity a definite, though somewhat idealized scenario: Consider a fast parton injected into infinitely extended nuclear matter by some highly localized process of space-time extent, $\left(Q_{0}^{2}\right)^{-1 / 2} \ll \Lambda^{-1}$. This primary parton then propagates through the nucleus and initiates a cascade or shower of secondary partons. The partons in the cascade can either radiate bremsstrahlung gluons or produce $q \bar{q}$-pairs, collide with partons of the nucleus (the medium), or absorb nuclear partons. I will call such an event a parton shower or parton cascade. I emphasize that I will explicitly distinguish between the shower partons on the one hand, and the nuclear partons on the other hand which initially are coherently bound in the wave function of the nucleus. Also, for the time being, I consider here the evolution of a single cascade and therefore only account for interactions of the cascade with the medium, but neglect possible interactions between simultaneously evolving cascades.

The prototype reaction of such a scenario is a proton-nucleus $(p A)$ collision at very 
high beam energy, where one may trigger on a high energy time-like quark or gluon that is produced at some point of time $t_{0}$ inside the heavy nucleus by a hard scattering, and single out the development of the resulting parton shower inside the nucleus with a sufficiently homogenous spatial density. For the description of such a parton cascade it is convenient to choose the nucleon-nucleon center-of-mass frame $\left(\mathrm{CM}_{N N}\right)$ [24] in which each nucleon has the same value of longitudinal momentum $P$,

$$
P_{z}^{(p)}=+P, \quad P_{z}^{(A)}=-A P, \quad \vec{P}_{\perp}^{(p)}=\vec{P}_{\perp}^{(A)}=\overrightarrow{0}
$$

with

$$
\sqrt{s}=\sqrt{4 A P^{2}+M_{N}^{2}\left(1+A^{2}\right)}, \quad \sqrt{s_{N N}} \equiv 2 P
$$

where $A$ is the nuclear mass number, $M_{N}$ the nucleon mass, and $P / M_{N} \gg 1$ is assumed. For example, at the RHIC (LHC) the maximum available beam energy implies $P / M_{N}>$ 100 (3000) even for $p+A u$ collisions. I will describe the longitudinal evolution of a parton shower along the shower axis (z-axis), which I define parallel to the direction of momentum of the initiating primary parton. It is convenient to parametrize the four-momenta $k \equiv k^{\mu}=$ $\left(E, k_{z}, \vec{k}_{\perp}\right)$ of the shower partons such that for the primary parton,

$$
k_{0}=\left(x_{0} P+\frac{Q_{0}^{2}}{2 x_{0} P} ; x_{0} P, \overrightarrow{0}\right),
$$

whereas for the $j^{\text {th }}$ secondary parton,

$$
k_{j}=\left(x_{j} P+\frac{Q_{j}^{2}+k_{\perp j}^{2}}{2 x_{j} P} ; x_{j} P, \vec{k}_{\perp j}\right),
$$

where $x_{j}=p_{z j} / P$ can be either positive or negative depending on the partons direction of propagation along the z-axis. Furthermore, $k_{\perp j}^{2}<Q_{j}^{2} \ll P^{2}$ is assumed, and all rest masses are neglected. It is important to realize that energy and momentum are independent variables, since one is dealing with off-shell particles of virtuality $Q^{2}>0$ with a continuous invariant mass distribution. The evolution of the parton system is then described by the change of parton number densities, which are defined as 


$$
a\left(x, k_{\perp}^{2} ; Q^{2}, t\right)=\int_{0}^{Q^{2}} d Q^{\prime 2} \frac{d N_{a}(t)}{d x d k_{\perp}^{2} d Q^{\prime 2}}, \quad\left(a \equiv q_{i}, \bar{q}_{i}, g ; i=1, \ldots, n_{f}\right)
$$

or, when integrated over transverse momentum,

$$
a\left(x, Q^{2}, t\right)=\int d k_{\perp}^{2} a\left(x, k_{\perp}^{2} ; Q^{2}, t\right)
$$

The relation between the parton number densities (5) to the single-particle phase-space distributions is expressed by the number of partons of type $a$ present at time $t$

$$
N_{a}(t)=\int d^{3} r \int d^{4} k F_{a}(E, \vec{k} ; t, \vec{r}) \equiv \int d^{4} k f_{a}(E, \vec{k} ; t)
$$

with $F_{a}$ denoting the corresponding phase-space density of off-shell partons with virtuality $Q^{2}=E^{2}-\vec{k}^{2}$ in the phase-space volume $d E d^{3} k d^{3} r$ around $k^{\mu}$ and $\vec{r}$ at time $t$, and $f_{a}$ in the second line representing the spatially integrated energy-momentum distribution.

In order to describe the time evolution of the parton densities (5) or (6) one has to relate the change of the dynamical variables $x$ and $Q^{2}$ with the laboratory time $t$ which plays the role of an external 'parameter' rather than being an intrinsic kinematical quantity. In Ref. [1] it was shown, using time-dependent perturbation theory, that for the case of a parton shower evolving in vacuum (i.e. by successive branchings only), one finds that the time scale for a branching chain, starting at $t_{0}=0$ with initial values $Q_{0}^{2}$ and $x_{0}$, is in the average given by

$$
t=\sum_{j=1}^{n} \frac{\left|x_{j}\right| P}{Q_{j}^{2}} \simeq \frac{|x| P}{Q^{2}}
$$

with $\left(z_{j}=x_{j} / x_{j-1}\right)$

$$
x=x_{n}=z_{1} z_{2} \ldots z_{n} x_{0}, \quad Q^{2}=Q_{n}^{2} \ll Q_{n-1}^{2} \ll \ldots \ll Q_{0}^{2},
$$

in accord with the uncertainty principle. Thus, in the absence of interactions with surrounding matter, the time variable $t$ is related to $x$ and and the degree of off-shellness $Q^{2}$ by the typical life-time $1 / Q$ in the parton's restframe, boosted by the Lorentz factor $\gamma=|x| P / Q$. However, when considering a parton shower inside nuclear matter, scattering and fusion 
processes with nuclear partons will compete with spontaneous branchings and consequently disrupt a steady decrease of average virtuality with time. Both scatterings and fusions can increase the virtuality of a parton by energy-momentum transfer. This means that the relation (9) between $x, Q^{2}$ and $t$ cannot be deterministic anymore, because interactions with the nuclear medium occur stochastically according to the density of nuclear partons. Each scattering or fusion may "rejuvenate" a shower parton by providing it with a virtuality $Q^{\prime 2}>Q^{2}$ so that the parton after the interaction appears to be younger $\left(t<t^{\prime}\right)$. To keep track of these repeated rejuvenations, one has to reset the clock for the particular shower parton after each such interaction with the medium. For this purpose a new independent dynamic variable $\tau$, called the age of a parton, was introduced in Ref. [1]. Instead of (9), one finds

$$
\tau \equiv \tau(x, t)=\left(\frac{|x| P}{Q^{2}}\right)_{t},
$$

which determines the typical age of a parton depending on $x, Q^{2}$ and implicitely also on $t$, and which introduces an additional time scale that reflects the external influence of the medium on the time evolution of the parton cascade. Correspondingly, the parton number densities (5) and (6) must be generalized as

$$
a(x, \tau, t)=\int_{\tau_{0}}^{\tau} d \tau^{\prime} \int d k_{\perp}^{2} \frac{d N_{a}(t)}{d x d k_{\perp}^{2} d \tau^{\prime}}
$$

which now has the meaning of the probability density for finding a parton of type $a$ at time $t$ with age $\tau=|x| P / Q^{2}$ and momentum fraction $x$. The age variable measures here the influence of the nuclear medium on the development of the parton cascade. Note that in absence of a background medium the age variable looses its independent character, i.e. it then evolves parallel to laboratory time and $\tau \propto t$. I will return to that point in Sec. 3 .

\subsection{The evolution equations}

For the remainder of this paper I will be concerned only with transverse momentum integrated quantities, so that it suffices to describe the evolution in terms of the parton number 
densities $q_{i},\left(\bar{q}_{i}\right)$ and $g$, defined by eq. (11). In addition, it is convenient to introduce the parton momentum densities $Q_{i}\left(\bar{Q}_{i}\right)$ and $G$

$$
Q_{i}(x, \tau, t) \equiv x q_{i}(x, \tau, t), \quad G(x, \tau, t) \equiv x g(x, \tau, t)
$$

i.e. the parton number densities weighted with the longitudinal momentum fraction $x$, and integrated over all transverse momenta $k_{\perp}^{2}$.

As graphically illustrated in Fig. 1, the coupled evolution equations for the parton densities $Q_{i}$ of quarks, $\bar{Q}_{i}$ of antiquarks, and $G$ of gluons, respectively, can be summarized now as follows [1]:

$$
\begin{aligned}
\left(\frac{\partial}{\partial t}+\frac{\partial}{\partial \tau}\right) Q_{i}(x, \tau, t)=- & {\left[\hat{A}+\hat{A}^{\prime}\{\tilde{G}\}+\hat{B}^{\prime}\left\{\tilde{\bar{Q}}_{i}\right\}\right] Q_{i}+\left[\hat{B}+\hat{S}_{q g}\left\{\tilde{Q}_{i}\right\}\right] G } \\
& +\sum_{j=1}^{n_{f}} \hat{S}_{q q}\left\{\tilde{Q}_{i}\right\} Q_{j}+\sum_{j=1}^{n_{f}} \hat{S}_{q \bar{q}}\left\{\tilde{Q}_{i}\right\} \bar{Q}_{j} \\
\left(\frac{\partial}{\partial t}+\frac{\partial}{\partial \tau}\right) \bar{Q}_{i}(x, \tau, t)=- & {\left[\hat{A}+\hat{A}^{\prime}\{\tilde{G}\}+\hat{B}^{\prime}\left\{\tilde{Q}_{i}\right\}\right] \bar{Q}_{i}+\left[\hat{B}+\hat{S}_{q g}\left\{\tilde{\bar{Q}}_{i}\right\}\right] G } \\
& +\sum_{j=1}^{n_{f}} \hat{S}_{q \bar{q}}\left\{\tilde{\bar{Q}}_{i}\right\} Q_{j}+\sum_{j=1}^{n_{f}} \hat{S}_{\bar{q} \bar{q}}\left\{\tilde{\bar{Q}}_{i}\right\} \bar{Q}_{j} \\
\left(\frac{\partial}{\partial t}+\frac{\partial}{\partial \tau}\right) G(x, \tau, t)=- & {\left[\hat{C}+\hat{D}+\hat{C}^{\prime}\left\{\tilde{G}^{\prime}\right\}+\sum_{j=1}^{n_{f}}\left\{\hat{D}^{\prime}\left\{\tilde{Q}_{j}\right\}+\hat{D}^{\prime}\left\{\tilde{\bar{Q}}_{j}\right\}\right\}-\hat{S}_{g g}\{\tilde{G}\}\right] } \\
& +\sum_{j=1}^{n_{f}}\left[\hat{E}+\hat{E}^{\prime}\left\{\tilde{\bar{Q}}_{j}\right\}+\hat{S}_{g q}\{\tilde{G}\}\right] Q_{j} \\
& +\sum_{j=1}^{n_{f}}\left[\hat{E}+\hat{E}^{\prime}\left\{\tilde{Q}_{j}\right\}+\hat{S}_{g \bar{q}}\{\tilde{G}\}\right] \bar{Q}_{j}
\end{aligned}
$$

Note that this set of equations describes the time evolution of the parton (longitudinal) momentum distributions $Q=x q, \bar{Q}=x \bar{q}$ and $G=x g$, rather than of the parton number densities $q, \bar{q}$ and $g$. The left hand side of these equations describes just the free streaming in the absence of interactions, whereas on the right hand side the various integral operators acting on the parton momentum densities desribe the change of the densities due to branching, fusion, and scattering processes. In contrast to the branching operators $(\hat{A}, \hat{B}, \ldots, \hat{E})$, the operators that describe fusions $\left(\hat{A}^{\prime}\{\tilde{Q}\}, \hat{B}^{\prime}\{\tilde{Q}\}, \ldots, \hat{E}^{\prime}\{\tilde{G}\}\right)$ and scattering processes $\left(\hat{S}_{q q}\{\tilde{Q}\}, \ldots, \hat{S}_{g g}\{\tilde{G}\}\right)$ are functionals of the nuclear parton distributions (labeled 
with a ${ }^{\prime}$ ') of quarks $\tilde{Q}(\tilde{\bar{Q}})$ and gluons $\tilde{G}$.

In order to represent the integral operators in the evolution equations (13)-(15) in a compact form, let me introduce effective coupling functions defined in terms of the running QCD coupling strength in one-loop order

$$
\alpha_{s}\left(Q^{2}\right)=\frac{12 \pi}{\left(33-2 n_{f}\right) \ln \left(Q^{2} / \Lambda^{2}\right)}
$$

where $\Lambda$ is the QCD renormalization scale and $n_{f}$ is the number of quark flavors that can be probed at scale $Q^{2}$. The following definitions will be employed:

$$
\begin{gathered}
\alpha_{s}(\tau) \equiv \frac{\int_{0}^{1} d x \alpha_{s}\left(\frac{|x| P}{\tau}\right) \theta\left(\frac{|x| P}{\tau}-\mu_{0}^{2}\right)\left(g(x, \tau, t)+\sum_{j}\left[q_{j}(x, \tau, t)+\bar{q}_{j}(x, \tau, t)\right]\right)}{\int_{0}^{1} d x\left(g(x, \tau, t)+\sum_{j}\left[q_{j}(x, \tau, t)+\bar{q}_{j}(x, \tau, t)\right]\right)} \\
\xi(\tau) \equiv \frac{\alpha_{s}(\tau)}{2 \pi \tau},\left.\quad \zeta(x, \tau) \equiv \frac{4 \pi \alpha_{s}\left(M^{2}\right)}{M^{2}}\right|_{M^{2}=|x| P / \tau} .
\end{gathered}
$$

Here $\alpha_{s}(\tau)$ describes the $x$-averaged QCD coupling at scale $Q^{2}=|x| P / \tau$, and the age $\tau$ represents the mean life-time of a parton with longitudinal momentum fraction $x$ and virtuality $Q^{2}$. Finally I denote by $\gamma_{a \rightarrow b c}(z)$ the usual branching functions [13, 14],

$$
\begin{aligned}
& \gamma_{q \rightarrow q g}(z)=C_{Q}\left(\frac{1+z^{2}}{1-z}\right) \\
& \gamma_{q \rightarrow g q}(z)=C_{Q}\left(\frac{1+(1-z)^{2}}{z}\right) \\
& \gamma_{g \rightarrow g g}(z)=2 C_{G}\left(z(1-z)+\frac{z}{1-z}+\frac{1-z}{z}\right) \\
& \gamma_{g \rightarrow q \bar{q}}(z)=\frac{1}{2}\left(z^{2}+(1-z)^{2}\right),
\end{aligned}
$$

where $C_{Q}=\frac{n_{c}^{2}-1}{2 n_{c}}=4 / 3, C_{G}=n_{c}=3$, and $z=x_{b} / x_{a}$ is the fraction of $x$-values of daughter to mother partons in the branching $a \rightarrow b c$.

As is evident from the evolutions equations (13)-(15), the rate of time change of the number of partons receives various contributions from the different interactions of the cascading partons, represented by their momentum densities $Q, \bar{Q}$ and $G$, from $1 \rightarrow 2$ branchings, $2 \rightarrow 1$ fusions and $2 \rightarrow 2$ scatterings. In addition to the well known parton evolution in 
vacuum (e.g. jet development in $e^{+} e^{-}$-annihilation), in which only spontanous branching processes contribute, in the present scenario of parton evolution in nuclear matter, the interactions with the medium, i.e. with the nuclear partons, give rise to fusion and scattering processes, and indirectly also to stimulated branchings. The physical picture underlying this probabilistic approach to generate such parton cascades from sequential elementary $1 \rightarrow 2$, $2 \rightarrow 1$ and $2 \rightarrow 2$ interactions is a direct generalization of the standard perturbative QCD parton shower picture 15,23]. For more details I refer to Ref. [1]. Here I will only list the corresponding expressions of the integral operators in eqs. (13)-(15) corresponding to the diagrams in Fig. 1.

\subsection{Branching processes}

For cascading quarks the $1 \rightarrow 2$ branching processes are balanced by the loss $(-)$ and gain $(+)$ rates

$$
\begin{aligned}
-\hat{A} Q_{i} & =-\int_{0}^{1} d z\left[Q_{i}(x, \tau, t)-Q_{i}\left(\frac{x}{z}, \tau, t\right)\right] \xi(\tau) \gamma_{q \rightarrow q g}(z) \\
+\hat{B} G & =+\int_{0}^{1} d z G\left(\frac{x}{z}, \tau, t\right) \xi(\tau) \gamma_{g \rightarrow q \bar{q}}(z) .
\end{aligned}
$$

The corresponding branching rates for antiquarks are obtained upon substitution $Q_{i} \leftrightarrow \bar{Q}_{i}$. The loss and gain branching rates of gluons are given by:

$$
\begin{aligned}
& -\hat{C} G=-\int_{0}^{1} d z\left[\frac{1}{2} G(x, \tau, t)-G\left(\frac{x}{z}, \tau, t\right)\right] \xi(\tau) \gamma_{g \rightarrow g g}(z) \\
& -\hat{D} G=-n_{f} G(x, \tau, t) \int_{0}^{1} d z \xi(\tau) \gamma_{g \rightarrow q \bar{q}}(z) \\
& +\hat{E} Q_{j}=+\int_{0}^{1} d z Q_{j}\left(\frac{x}{z}, \tau, t\right) \xi(\tau) \gamma_{q \rightarrow q g}(z) \\
& +\hat{E} \bar{Q}_{j}=+\int_{0}^{1} d z \bar{Q}_{j}\left(\frac{x}{z}, \tau, t\right) \xi(\tau) \gamma_{q \rightarrow q g}(z) .
\end{aligned}
$$

\subsection{Fusion processes}

The $2 \rightarrow 1$ fusion rates that describe the effective loss of quarks due to fusions are:

$$
-\hat{A}^{\prime}\{\tilde{G}\} Q_{i}=-\rho_{N} c_{q g \rightarrow q} \int_{0}^{1} d z\left[Q_{i}(x, \tau, t) \tilde{G}\left(\frac{x(1-z)}{z}\right) \zeta\left(\frac{x}{z}, \tau\right)\right.
$$




$$
\begin{gathered}
\left.-Q_{i}(x z, \tau, t) \tilde{G}(x(1-z))\right] \gamma_{q \rightarrow q g}(z) \zeta(x, \tau) \\
-\hat{B}^{\prime}\left\{\tilde{\bar{Q}}_{i}\right\} Q_{i}=-\rho_{N} c_{q \bar{q} \rightarrow g} \int_{0}^{1} d z Q_{i}(x, \tau, t) \tilde{\bar{Q}}_{i}\left(\frac{x(1-z)}{z}\right) \gamma_{g \rightarrow q \bar{q}}(z) \zeta\left(\frac{x}{z}, \tau\right) .
\end{gathered}
$$

Here $\rho_{N}$ is the nucleon density of the nuclear medium (to be specified below), and the constants $c_{q g \rightarrow q}=c_{\bar{q} g \rightarrow \bar{q}}=1 / 8, c_{q \bar{q} \rightarrow g}=8 / 9$ arise from the difference of flux factors (color and spin) for fusions compared to branchings [25]. Similarly the fusion rates of antiquarks are given by replacing $Q_{i} \leftrightarrow \bar{Q}_{i}$. The corresponding loss and gain fusion rates of gluons are:

$$
\begin{array}{r}
-\hat{C}^{\prime}\{\tilde{G}\} G=-\rho_{N} c_{g g \rightarrow g} \int_{0}^{1} d z\left[G(x, \tau, t) \tilde{G}\left(\frac{x(1-z)}{z}\right) \zeta\left(\frac{x}{z}, \tau\right)\right. \\
-G(x z, \tau, t) \tilde{G}(x(1-z)) \zeta(x, \tau)] \gamma_{g \rightarrow g g}(z) \\
-\hat{D}^{\prime}\left\{\tilde{Q}_{j}\right\} G=-\rho_{N} c_{g q \rightarrow q} G(x, \tau, t) \int_{0}^{1} d z \tilde{Q}_{j}\left(\frac{x(1-z)}{z}\right) \gamma_{q \rightarrow g q}(z) \zeta\left(\frac{x}{z}, \tau\right) \\
-\hat{D}^{\prime}\left\{\tilde{\bar{Q}}_{j}\right\} G=-\rho_{N} c_{g q \rightarrow q} G(x, \tau, t) \int_{0}^{1} d z \tilde{\bar{Q}}_{j}\left(\frac{x(1-z)}{z}\right) \gamma_{q \rightarrow g q}(z) \zeta\left(\frac{x}{z}, \tau\right) \\
+\hat{E}^{\prime}\left\{\tilde{\bar{Q}}_{j}\right\} Q_{j}=+\frac{1}{2} \rho_{N} c_{q \bar{q} \rightarrow g} \int_{0}^{1} d z Q_{j}(x z) \tilde{\bar{Q}}_{j}(x(1-z)) \gamma_{g \rightarrow q \bar{q}}(z) \zeta(x, \tau) \\
+\hat{E}^{\prime}\left\{\tilde{Q}_{j}\right\} \bar{Q}_{j}=+\frac{1}{2} \rho_{N} c_{q \bar{q} \rightarrow g} \int_{0}^{1} d z \bar{Q}_{j}(x z) \tilde{Q}_{j}(x(1-z)) \gamma_{g \rightarrow q \bar{q}}(z) \zeta(x, \tau) .
\end{array}
$$

The flux factors are here $c_{g g \rightarrow g}=1 / 8, c_{g q \rightarrow q}=c_{g \bar{q} \rightarrow \bar{q}}=1 / 8, c_{q \bar{q} \rightarrow g}=8 / 9$.

In (21) and (22) the argument $x$ of the nuclear parton distributions $\tilde{Q}, \tilde{\bar{Q}}$ and $\tilde{G}$ is understood as $|x|$, since the nuclear partons move in $-P$ direction [c.f. eq. (1)], but the measured nucleon structure functions are usually defined only for positive calues of $x$. Also note that in (22) the second term on the right in the expression for $\hat{C}^{\prime}\{\tilde{G}\} G$ would be multiplied by a factor $1 / 2$ if $\tilde{G}$ and $G$ would be treated on equal footing, because nuclear and shower gluons would then be indistinguishable.

\subsection{Scattering processes}

The $2 \rightarrow 2$ collision rates for elastic scatterings of the cascading partons carrying momentum fractions $x_{1}$ and $x_{2}$ with the partons in the nuclear background medium of nucleon density $\rho_{N}$ carrying momentum fractions $x_{1}^{\prime}$ and $x_{2}^{\prime}$ are represented by the integral operator

$$
\hat{S}_{a b}\{\tilde{A}\} B \equiv-\int_{-1}^{0} \frac{d x_{2}}{x_{2}} \tilde{A}\left(x_{2}\right) \int d p_{\perp}^{2} \frac{d \hat{\sigma}_{a b \rightarrow a b}}{d p_{\perp}^{2}} \rho_{N} \frac{B(x, \tau, t)}{x}
$$




$$
\begin{aligned}
& +\left.\frac{|x| P}{\tau^{2}} \int_{-1}^{0} \frac{d x_{2}}{x_{2}} \tilde{A}\left(x_{2}\right) \frac{d \hat{\sigma}_{a b \rightarrow a b}}{d p_{\perp}^{2}}\right|_{p_{\perp}^{2}=\frac{|x| P}{\tau}} \\
& \quad \times \rho_{N} \int_{0}^{\infty} d \tau_{1} \frac{B\left(x_{1}, \tau_{1}, t\right)}{x_{1}}\left[1+\theta\left(\frac{\tau_{1}}{\tau}-\frac{x_{1}}{|x|}\right)\right] \\
& +\int_{-1}^{0} \frac{d x_{2}}{x_{2}} \tilde{A}\left(x_{2}\right) \int^{x_{1} P / \tau} d p_{\perp}^{2} \frac{d \hat{\sigma}_{a b \rightarrow a b}}{d p_{\perp}^{2}} \rho_{N} \frac{B\left(x_{1}, \tau, t\right)}{x_{1}},
\end{aligned}
$$

where $A, B=G, Q_{j}, \bar{Q}_{j}$ and the " ' labels as before the nuclear parton distributions, whereas the distributions without ' , refer to the cascading partons. As in the case of fusions, eqs. (21) and (22), the value of $x_{2}$ in the argument of the nuclear parton distribution $\tilde{A}$ is meant as $\left|x_{2}\right|$ when employing standard parametrizations of nucleon structure functions.

The momentum fractions of two partons before $\left(x_{1}, x_{2}\right)$ and after $\left(x_{1}^{\prime}, x_{2}^{\prime}\right)$ scattering involving a relative transverse momentum exchange $p_{\perp}^{2}$ are related by

$$
x_{1}=x_{1}^{\prime}+\frac{p_{\perp}^{2}}{\left(x_{1}^{\prime}-x_{2}\right) P^{2}} \quad, \quad x_{2}=x_{2}^{\prime}+\frac{p_{\perp}^{2}}{\left(x_{2}^{\prime}-x_{1}\right) P^{2}},
$$

from which follows the value of $x_{1}$ at which the function $B\left(x_{1}, \tau, t\right)$ in eq. (23) is to be evaluated,

$$
x_{1}=x+\frac{p_{\perp}^{2}}{\left(x-x_{2}\right) P^{2}} .
$$

For the quarks (and similar antiquarks) one has the processes $q_{i} g \rightarrow q_{i} g, q_{i} q_{j} \rightarrow q_{i} q_{j}$ and $q_{i} \bar{q}_{j} \rightarrow q_{i} \bar{q}_{j}$, whereas for gluons the contributing processes are $g g \rightarrow g g, g q_{j} \rightarrow g q_{j}$ and $g \bar{q}_{j} \rightarrow g \bar{q}_{j}$. The parton-parton cross-sections $d \hat{\sigma}_{a b \rightarrow c d} / d p_{\perp}^{2}$ in the scattering function (23) are related to the squared scattering amplitudes $\left|\overline{\mathcal{M}}_{a b \rightarrow c d}\right|^{2}$, averaged over initial spin and color states and summed over the final states, by (neglecting the quark masses),

$$
\frac{d \hat{\sigma}_{a b \rightarrow c d}\left(\hat{s}, p_{\perp}^{2}\right)}{d p_{\perp}^{2}}=\mathcal{D}_{a b} \mathcal{D}_{c d} \frac{\pi \alpha_{s}^{2}\left(p_{\perp}^{2}\right)}{\hat{s}^{2}}\left|\overline{\mathcal{M}}_{a b \rightarrow c d}\right|^{2}
$$

where the degeneracy factors $\mathcal{D}_{a b}=\left(1+\delta_{a b}\right)^{-1}$ accounts for the identical particle effect in the initial state if $a$ and $b$ are truly indistinguishable, and correspondingly $\mathcal{D}_{c d}$ is the statistical factor for the final state. However, since cascading particles and nuclear partons are assumed to be distinguishable, one has $\mathcal{D}_{a b}=1$ always (but not so for $\mathcal{D}_{c d}$ ). Since needed later, let me list for completeness the relevant squared matrix elements for the various processes [26]: 


$$
\begin{aligned}
\left|\overline{\mathcal{M}}_{q_{i} q_{j} \rightarrow q_{i} q_{j}}\right|^{2} & =\frac{4}{9}\left(\frac{\hat{s}^{2}+\hat{u}^{2}}{\hat{t}^{2}}\right)+\delta_{i j}\left[\frac{4}{9}\left(\frac{\hat{s}^{2}+\hat{t}^{2}}{\hat{u}^{2}}\right)-\frac{8}{27}\left(\frac{\hat{s}^{2}}{\hat{u} \hat{t}}\right)\right] \\
\left|\overline{\mathcal{M}}_{q_{i} \bar{q}_{j} \rightarrow q_{i} \bar{q}_{j}}\right|^{2} & =\frac{4}{9}\left(\frac{\hat{s}^{2}+\hat{u}^{2}}{\hat{t}^{2}}\right)+\delta_{i j}\left[\frac{4}{9}\left(\frac{\hat{t}^{2}+\hat{u}^{2}}{\hat{s}^{2}}\right)-\frac{8}{27}\left(\frac{\hat{u}^{2}}{\hat{s} \hat{t}}\right)\right] \\
\left|\overline{\mathcal{M}}_{q_{i} g \rightarrow q_{i} g}\right|^{2} & =-\frac{4}{9}\left(\frac{\hat{u}^{2}+\hat{s}^{2}}{\hat{u} \hat{s}}\right)+\left(\frac{\hat{u}^{2}+\hat{s}^{2}}{\hat{t}^{2}}\right) \\
\left|\overline{\mathcal{M}}_{g g \rightarrow g g}\right|^{2} & =\frac{9}{2}\left(3-\frac{\hat{u} \hat{t}}{\hat{s}^{2}}-\frac{\hat{u} \hat{s}}{\hat{t}^{2}}-\frac{\hat{s} \hat{t}}{\hat{u}^{2}}\right),
\end{aligned}
$$

The variables $\hat{s}, \hat{t}, \hat{u}$ are the kinematic invariants of the parton-parton scattering with $\hat{s}+\hat{t}+\hat{u}=0$, and $p_{\perp}^{2}=\hat{t} \hat{u} / \hat{s}$ for massless particles. For massive quarks the corresponding scattering matrix-elements can be found in Ref. [27].

\section{ANALYTICAL SOLUTIONS FOR THE PARTON SHOWER FUNCTIONS}

In the following I will present a method for solving the evolution equations (13)-(15) analytically and will investigate some characteristic features of the solutions. Recall the scenario from Sec. 2 with a highly energetic incident quark or gluon, produced at some point of time $t_{0}$ inside the target nucleus, with longitudinal momentum fraction $x_{0} \simeq 1$, transverse

momentum $\vec{k}_{\perp 0}=\overrightarrow{0}$, and virtuality $Q_{0}^{2} \gg \Lambda$, that initiates a cascade (or shower) of secondary quarks, antiquarks, and gluons. By solving the evolution equations, one can follow the time evolution of this parton shower in the nuclear background medium consisting of the partons of the heavy nucleus. In the present section I will solve the evolution equations (13)-(15) for the parton momentum densities $Q(\bar{Q})$ and $G$, or equivalently the number densities $q(\bar{q})$ and $g$. The synonym parton shower functions as generic term for these distributions will be frequently used. As I will show, the evolution equations can be solved in closed form, if one restricts to a somewhat idealized scenario that allows for a transparent analysis (without however giving up the physical relevance of application to realistic situations).

\subsection{Assumptions and approximations}

The assumptions and approximations that I will have to impose in order to achieve tractable solutions are the following: 
(i) As before I will employ a rigorous distinction between the cascading partons produced by the evolving parton shower and the partons of the nucleus which represent the medium. Only the shower partons are followed dynamically. The nuclear parton distributions are approximated by a constant behaviour, motivated by a rather weak $x$-dependence of the nucleon structure functions at small values of $x$ [28]. I will restrict the shower development to the small $x$-region where the probability for an interaction of a parton is the largest, because of the logarithmically large integration over the momenta of the involved partons and the large available phase-space.

(ii) The nuclear background medium is assumed to be of very large (ideally of infinite) size with a uniform spatial distribution of nuclear partons, so that one can ignore boundary effects and average over the spatial coordinates. Also, I make the so-called "sudden approximation", i.e. the nuclear parton distributions remain essentially unaltered by the interactions with the shower partons, at least on time scales characteristic for the "intra-cascade activity". However, once a nuclear parton has interacted with a shower parton, it is considered to be materialized from the coherent medium and becomes part of the evolving cascade. Thus the medium acts as a particle and energy-momentum reservoir.

(iii) For the nuclear partons of the medium, I will ignore effects as nuclear shadowing, color coherence, long range correlations, etc., that affect the momentum and number distributions of those quanta. I approximate the nuclear distributions as simple superposition of the experimentally measured nucleon structure functions and consider the nuclear parton density as a function of the number of nucleons $A$ per unit area. The contributions of valence quarks are neglected, that is, the matter is taken to be effectively baryon free, an assumption that is reasonable for the valence quark depleted small $x$-region.

Accordingly, the attention is restricted here to the diffusion of cascading partons in longitudinal direction and the dissipation of longitudinal momentum (or energy) of the 
shower particles. That is, I will solve for the time evolution of the parton shower functions as a function of longitudinal momentum fraction $x$ and age $\tau$. A more realistic evolution including also the diffusion in transverse momentum $\vec{k}_{\perp}$, the lateral spread in $\vec{r}_{\perp}$, as well as equal treatment of shower and nuclear partons, requires a numerical analysis, which will be addressed in a separate work.

\subsection{Simplified form of the evolution equations}

Due to the dependence of the integral operators for the fusion processes and the scattering processes on the parton distributions $\tilde{Q}, \tilde{\bar{Q}}$, and $\tilde{G}$ of the nuclear background medium, the right hand side of the evolution equations involves quadratic forms of the parton distributions, because of the coupling of the shower partons to the nuclear partons. A general analytical solution of this non-linear problem is therefore not possible. To overcome this obstacle, I explicitely distinguish these two sources [c.f. item (i) above]. I approximate the nuclear parton densities as scale $\left(Q^{2}\right)$ independent and neglect their time variation, as well as nuclear shadowing effects, etc., [c.f. item (ii)] and simply represent them by the nucleon structure functions multiplied with a nuclear factor,

$$
\tilde{Q}_{i}(x)=\rho_{N} x \tilde{q}_{i}\left(|x|, Q_{0}^{2}\right), \quad \tilde{G}(x)=\rho_{N} x \tilde{g}\left(|x|, Q_{0}^{2}\right)
$$

where $\tilde{q}_{i}, \tilde{g}$ denote the usual (time-independent) measured nucleon structure functions, in principle to be evaluated at the initial scale $Q_{0}^{2}$ at which the primary parton is produced. It is important to realize that in (28) the values of $x=p_{z} / P<0$ in accordance with eq. (看), i.e. the fact that the nuclear partons move in -z-direction. Therefore, although the number densities $\tilde{q}_{i}, \tilde{g}$ are (as probability densities) necessarily always positive definite, the

momentum densities are here $\tilde{Q}_{i}, \tilde{G}<0$. The nuclear factor $\rho_{N}$ depends on the geometry and the nucleon density [25,29],

$$
\rho_{N}=\frac{3}{2} R_{A} \bar{n}_{N}
$$

where $R_{A}=r_{0} A^{1 / 3}, r_{0}=1.2 \mathrm{fm}$, and $\bar{n}_{N}=A /\left(4 \pi R_{A}^{3} / 3\right)$ are the nuclear radius and the nucleon density of the traversed nucleus, respectively. Furthermore, motivated by the 
aforementioned weak $x$-dependence of the nucleon structure functions at small values of $x \lesssim 10^{-2}$ (see however [28]), one may approximate the nuclear parton distributions (28) by a constant $\tilde{Q}^{0}$, respectively $\tilde{G}^{0}$, times the nuclear factor (29):

$$
\tilde{Q}_{i}(x)=\tilde{\bar{Q}}_{i}(x)=-\frac{9}{8 \pi} \frac{A^{1 / 3}}{r_{0}^{2}} \eta_{i} \tilde{Q}^{0}, \quad \tilde{G}(x)=-\frac{9}{8 \pi} \frac{A^{1 / 3}}{r_{0}^{2}} \tilde{G}^{0} .
$$

Here the overall minus sign ensures that the nuclear momentum densities are negative definite, as explained after eq. (28). These $x$ - and $t$-independent parametrizations mimic in a crude approximation the effective number of nuclear partons per unit area, weighted with their longitudinal momentum, as 'seen' by the evolving shower partons. Since at small $x$ the contribution of the valence quarks is negligable, the distributions $Q(\bar{Q})$ are understood to represent solely the sea quarks (sea antiquarks) for which I take $n_{f}=3$, that is, I account for the flavors $i=1,2,3 \equiv u, d, s$ with the relative proportions

$$
\eta_{i}=\left\{\begin{array}{rl}
1 & \text { for } i=u(\bar{u}) \\
1 & \text { for } i=d(\bar{d}) \\
0.43 & \text { for } i=s(\bar{s})
\end{array} .\right.
$$

As reasonable values for $\tilde{Q}^{0}$ and $\tilde{G}^{0}$ one may take:

$$
\tilde{Q}^{0} \approx 0.2, \quad \tilde{G}^{0} \approx 3
$$

As long one is concerned with color and flavor insensitive kinetics of the parton evolution, it is sufficient and convenient to consider $n_{f}=3$ identical types of quarks and antiquarks and the only flavor dependence comes from the relative quark admixtures (31) in the nuclear medium. Due to the absence of valence quarks the baryon number is zero and particleantiparticle symmetry implies $Q_{i}=\bar{Q}_{i}$. Therefore it is convenient to define the flavor singlet combination by the sum over all quark flavors,

$$
\mathcal{Q}:=\sum_{i=1}^{n_{f}}\left(Q_{i}+\bar{Q}_{i}\right)
$$

and consider quarks and corresponding antiquarks as one particle species. 
Collecting all these ingredients, one can now rewrite the evolution equations (13)-(15) in a much simpler, linear form:

$$
\begin{aligned}
\frac{\partial}{\partial t} \mathcal{Q}(x, \tau, t)= & -\left[\hat{A}+\hat{A}^{\prime}\left\{\tilde{G}^{0}\right\}+\frac{1}{2} \hat{B}^{\prime}\left\{\kappa \tilde{Q}^{0}\right\}-n_{f} \hat{S}_{q q}\left\{\kappa \tilde{Q}^{0}\right\}+\frac{\partial}{\partial \tau}\right] \mathcal{Q} \\
& +\left[2 \hat{B}+\hat{S}_{q g}\left\{\kappa \tilde{Q}^{0}\right\}\right] G \\
\equiv & -\hat{F}_{q q} \mathcal{Q}+\hat{F}_{q g} G \\
\frac{\partial}{\partial t} G(x, \tau, t)= & -\left[\hat{C}+\hat{D}+\hat{C}^{\prime}\left\{\tilde{G}^{0}\right\}+n_{f} \hat{D}^{\prime}\left\{\kappa \tilde{Q}^{0}\right\}-\hat{S}_{g g}\left\{\tilde{G}^{0}\right\}+\frac{\partial}{\partial \tau}\right] G \\
& +\left[\hat{E}+\frac{1}{2} \hat{E}^{\prime}\left\{\kappa \tilde{Q}^{0}\right\}+\hat{S}_{g q}\left\{\tilde{G}^{0}\right\}\right] \mathcal{Q} \\
\equiv & -\hat{F}_{g g} G+\hat{F}_{g q} \mathcal{Q} .
\end{aligned}
$$

Here $\eta_{i}$ is defined by (31) and the constant $\kappa$ accounts for the effective number of quark flavors in the nuclear medium that are probed by the cascading gluons,

$$
\kappa:=\frac{1}{n_{f}} \sum_{i=1}^{n_{f}} \eta_{i}=0.81 .
$$

In (34) and (35) both the parton densities and the integral operators depend still on the three variables $t, \tau$, and $x$, corresponding to the time development of the vituality (age) distribution and energy (longitudinal momentum) distribution, respectively. For each age $\tau=|x| P / Q^{2}$ one must in general expect a different $x$-dependence (and in principle also flavor dependence), reflected by an "age distribution" $\mathcal{A}_{a}(\tau, t ; x)$ which represents the probability that a parton $a=q, g$ with $x$ at time $t$ has an age $\tau$, and which, for quarks and gluons, respectively, is defined through [1]

$$
\mathcal{Q}(x, \tau, t)=\mathcal{A}_{q}(\tau, t ; x) \mathcal{Q}(x, t), \quad G(x, \tau, t)=\mathcal{A}_{g}(\tau, t ; x) G(x, t)
$$

with normalization $\int_{0}^{\infty} d \tau \mathcal{A}_{a}(\tau, t ; x)=1$, and

$$
\mathcal{A}_{a}(\tau, t) \equiv \int_{0}^{1} d x \mathcal{A}_{a}(\tau, t ; x), \quad \bar{f}(t) \equiv \int_{0}^{\infty} d \tau \int_{0}^{1} d x \mathcal{A}_{a}(\tau, t ; x) f(x, \tau, t)
$$

The age distribution evolves parallel with laboratory time in between interactions (free streaming), but due to scatterings it receives modifications because the age of the scattered 
parton is reset to a younger age corresponding to a larger virtuality caused by the momentum transfer of the interaction. In the absence of a medium $\mathcal{A}=\delta(t-\tau)$. Therefore the age distribution reflects the influence of the nuclear medium and determines the characteristic age $\tau$. The age $\tau$ must hence be related to the mean free time in between scatterings and consequently introduces an external scale depending on the density of the medium. The separation of the medium influence in the parton densities by introducing $\mathcal{A}$, suggests to describe the parton evolution by the time rate of change with respect to the variable $x$ only and interpret the age distribution as externally induced feedback to the shower functions via (37) and (34), (35). Therefore let me rewrite the latter evolution equations in terms of $\mathcal{Q}(x, t), G(x, t)$ and $\mathcal{A}(\tau, t)$, defined in (38), by substituting the factorized forms (37):

$$
\begin{aligned}
\frac{\partial}{\partial t} \mathcal{Q}(x, t) & =-\left\{\hat{F}_{q q}+\frac{1}{\mathcal{A}}\left(\frac{\partial}{\partial t}+\frac{\partial}{\partial \tau}\right) \mathcal{A}\right\} \mathcal{Q}+\hat{F}_{q g} G \\
\frac{\partial}{\partial t} G(x, t) & =\hat{F}_{g q} \mathcal{Q}-\left\{\hat{F}_{g g}+\frac{1}{\mathcal{A}}\left(\frac{\partial}{\partial t}+\frac{\partial}{\partial \tau}\right) \mathcal{A}\right\} G .
\end{aligned}
$$

Here the terms involving $\mathcal{A}$ must in reality determined selfconsistently by the dynamic interplay between the shower particles and the nuclear environment. It contains implicitely information about the nuclear structure which at this point has to be provided as phenomenological input. However, the actual form of $\mathcal{A}$ is irrelevant if one is not interested in the age distribution itself, but rather is concerned with the time evolution of the partons with respect to $x$ and average $\tau$. In this case one may multiply both sides of the equations by $\mathcal{A}$, integrate over $\tau$ subject to the conditions (38), so that the explicit dependence on $\mathcal{A}$ drops out. Then one is left with eqs. (39), (40), now without the terms $\mathcal{A}^{-1}(\partial / \partial t+\partial / \partial \tau) \mathcal{A}$ (taking $\mathcal{A}_{q} \approx \mathcal{A}_{g}$ ), but one has to replace everywhere in the operators $\hat{F}_{a b}$

$$
\tau \longrightarrow \bar{\tau}(t)=v_{\tau}(t) t \equiv \int_{0}^{\infty} d \tau \tau \mathcal{A}(\tau, t)
$$

where

$$
v_{\tau}(t)=\frac{d \bar{\tau}(t)}{d t} \leq 1
$$

is the "velocity" by which the typical $\bar{\tau}$ changes with laboratory time $t$. The evolution equations can now be written in a compact form [15]: If one represents the time evolving 
parton distributions in a symbolic matrix form as $\hat{U}\left(t, t_{0}=0\right)=\exp [-i \hat{H} t]$ with $\hat{H}$ the Hamiltonian of the system, then the evolving parton state will form a vector $(\mathcal{Q}, G)$ which satisfies the following Schrödinger type equation

$$
\frac{\partial}{\partial t}\left(\begin{array}{l}
\mathcal{Q} \\
G
\end{array}\right)=-i \hat{H}\left(\begin{array}{l}
\mathcal{Q} \\
G
\end{array}\right)=\left(\begin{array}{cc}
-\hat{F}_{q q} & \hat{F}_{q g} \\
\hat{F}_{g q} & -\hat{F}_{g g}
\end{array}\right)\left(\begin{array}{l}
\mathcal{Q} \\
G
\end{array}\right) .
$$

As I will now show, this matrix equation, or equivalently the simplified evolution equations (39) and (40) averaged over $\tau$ according to (41), can be solved in closed form by diagonalizing the Hamiltonian $\hat{H}$, subject to some chosen initial condition at $t=t_{0}$.

\subsection{Solutions for the parton evolution}

The integral operators in the matrix equation (43) are now forms of the average age $\bar{\tau}(t)$ and the longitudinal momentum fractions $x=p_{z} / P$ of the shower particles only. As stated before, the time-dependent $\bar{\tau}(t)$ loosely speaking reflects the structure of the nuclear medium as probed by the shower partons, and it reduces in free space to the trivial dependence $\bar{\tau}=t$, i.e. $v_{\tau}=1$ in (42). Thus, one qualitatively expects a delayed parton evolution in medium as compared to vacuum, because repeated rejuvenations of the shower partons must lead to $\bar{\tau}<t$, or $v_{\tau} \leq 1$. If $v_{\tau}(t)$ has only a weak time-dependence, $v_{\tau} \approx$ const., then an appropriate solution ansatz for the parton shower functions is the following factorized form with a power dependence in $1 / x$ and an exponential decay with some time-dependent function $\chi(t)$ :

$$
\begin{aligned}
& \mathcal{Q}(x, t)=\int_{0}^{\infty} d \tau \mathcal{A}_{q}(\tau, t) \mathcal{Q}(x, \tau, t)=\mathcal{N}_{q}\left(|x|^{-s} \theta(x)+|x|^{s} \theta(-x)\right) \exp [-\mu \chi(t)] \\
& G(x, t)=\int_{0}^{\infty} d \tau \mathcal{A}_{g}(\tau, t) G(x, \tau, t)=\mathcal{N}_{g}\left(|x|^{-s} \theta(x)+|x|^{s} \theta(-x)\right) \exp [-\mu \chi(t)]
\end{aligned}
$$

Let me explain the physical motivation of this ansatz:

(i) The introduced power $s$ is understood to be positive definite, $s \geq 0$, and the constants $\mathcal{N}_{q}$ and $\mathcal{N}_{g}$ are normalization factors. As will become clear, the 'parameter' $s$ plays the role of a conjugate variable to the variable $x$, controlling the longitudinal momentum distribution in $x$. As the shower evolves, $s$ will change with time, thereby correlating the $x$ and the $t$ dependence of the shower functions. 
(ii) The form of the $x$-dependence in brackets is motivated by the particular dynamics viewed in the $C M_{N N}$ frame (Sec. 2.1) in which one naturally expects some "drag effect" of the (in $-z$ direction moving) nuclear partons on the evolving parton shower. Although the shower partons initially propagate in $+z$ direction, one must account at some point for particles moving in both $+z$ and $-z$ direction. Shower partons moving in $+z$ direction will progressively degrade their longitudinal momentum by branchings and scatterings, and will eventually reverse their longitudinal direction, dragged along and accelerated in $-z$ direction by absorption of (fusions with) comoving nuclear partons.

(iii) The exponential factor accounts for the fact that the running coupling strength $\alpha_{s}$ in the operators $\hat{F}_{a b}$ of (43) depends on $\bar{\tau}(t)$ and therefore implicitely on $t$. By introducing the function

$$
\chi(t) \equiv \int_{t_{0}}^{t} d t^{\prime} \frac{\alpha_{s}\left(\bar{\tau}\left(t^{\prime}\right)\right)}{2 \pi \bar{\tau}\left(t^{\prime}\right)}=\frac{1}{2 \pi b v_{\tau}} \ln \left(\frac{\alpha_{s}\left(v_{\tau} t\right)}{\alpha_{s}\left(v_{\tau} t_{0}\right)}\right),
$$

where $b=\left(11 n_{c}-2 n_{f}\right) / 12 \pi=0.72$ and $\bar{\tau}=v_{\tau} t$ with $v_{\tau}$ assumed to be only slowly varying with $t$, it is possible to absorb the medium dependent factor $\alpha_{s}(\bar{\tau}) /(2 \pi \bar{\tau})$ in the exponential time dependence of the distributions (44). Note that for fixed coupling and $v_{\tau}=$ const., one has $\chi \propto \ln \left(t / t_{0}\right)$. Even in the general case $\chi$ represents approximately the logarithm of the laboratory time $t$. The real time dependence in the laboratory frame is regained by the inverse relation

$$
t=\left(\frac{x P}{v_{\tau} \Lambda^{2}}\right) \exp \left[-\ln \left(\frac{Q_{0}^{2}}{\Lambda^{2}}\right) \exp \left(-2 \pi b v_{\tau} \chi\right)\right],
$$

where $Q_{0}^{2}$ is the virtuality of the shower initiating parton at $t_{0}$.

Substituting the ansatz (44) into the time-evolution equation (43), one arrives at the following eigenvalue equations with respect to the new time evolution variable $\chi(t)$, corresponding to the diagonalized Hamiltonian $\hat{H}$ : 


$$
\frac{\partial}{\partial \chi}\left(\begin{array}{l}
\mathcal{Q} \\
G
\end{array}\right)=-\mu\left(\begin{array}{l}
\mathcal{Q} \\
G
\end{array}\right)
$$

or equivalently

$$
\begin{aligned}
\mu & =F_{q q}(s)-\frac{b}{a} F_{q g}(s) \\
\frac{b}{a} \mu & =\frac{b}{a} F_{g g}(s)-F_{g q}(s) .
\end{aligned}
$$

Here the scalar functions $F_{a b}(s)$ correspond to the operators in the Hamiltonian, $\hat{F}_{a b}$, defined by (34) and (35),

$$
\begin{aligned}
& F_{q q}(s)=A(s)+A_{\left\{\tilde{G}^{0}\right\}}^{\prime}(s)+\frac{1}{2} B_{\left\{\kappa \tilde{Q}^{0}\right\}}^{\prime}(s)-n_{f} S_{\left\{\kappa \tilde{Q}^{0}\right\}}^{(q q)}(s) \\
& F_{q g}(s)=2 B(s)+S_{\left\{\kappa \tilde{Q}^{0}\right\}}^{(q g)}(s) \\
& F_{g q}(s)=n_{f} \hat{E}+n_{f} E_{\left\{\kappa \tilde{Q}^{0}\right\}}^{\prime}(s)+n_{f} S_{\left\{\tilde{G}^{0}\right\}}^{(g q)}(s) \\
& F_{g g}(s)=C(s)+C_{\left\{\tilde{G}^{0}\right\}}^{\prime}(s)+D(s)+n_{f} D_{\left\{\kappa \tilde{Q}^{0}\right\}}^{\prime}(s)-S_{\left\{\tilde{G}^{0}\right\}}^{(g g)}(s) .
\end{aligned}
$$

The branching kernels in (49) are

$$
\begin{aligned}
A(s) & =\int_{0}^{1} d z\left(1-z^{s}\right) \gamma_{q \rightarrow q g}(z)=C_{Q}\left\{2 \psi(s+2)+2 \gamma_{E}-\frac{1}{(s+1)(s+2)}-\frac{3}{2}\right\} \\
B(s) & =\int_{0}^{1} d z z^{s} \gamma_{g \rightarrow q \bar{q}}(z)=\frac{1}{2}\left\{\frac{s^{2}+3 s+4}{(s+1)(s+2)(s+3)}\right\} \\
C(s)+D(s) & =\int_{0}^{1} d z\left(\frac{1}{2}-z^{s}\right) \gamma_{g \rightarrow g g}(z)+n_{f} \int_{0}^{1} d z \gamma_{g \rightarrow q \bar{q}}(z) \\
& =C_{G}\left\{2 \psi(s+2)+2 \gamma_{E}-4 \frac{s^{2}+3 s+3}{s(s+1)(s+2)(s+3)}-\frac{11}{6}+\frac{n_{f}}{3 C_{G}}\right\} \\
E(s) & =\int_{0}^{1} d z z^{s} \gamma_{q \rightarrow g q}(z)=C_{Q} \frac{s^{2}+3 s+4}{s(s+1)(s+2)},
\end{aligned}
$$

where the branching functions $\gamma_{a \rightarrow b c}(z)$ are given by (18), and as before $n_{f}=3, C_{Q}=\frac{n_{c}^{2}-1}{2 n_{c}}$, $C_{G}=n_{c}$ with $n_{c}=3$. The Psi (Digamma) function is defined as $\psi(z)=d[\ln \Gamma(z)] / d z$ and $\gamma_{E}=0.5772$ is the Euler constant. Note that these branching kernels are finite, i.e. free of infrared singularities, because the infrared divergent contributions cancel explicitely due to the balance of gain and loss terms.

Similarly the fusion kernels are evaluated as: 


$$
\begin{aligned}
A_{\left\{\tilde{G}^{0}\right\}}^{\prime}(s)= & -\tilde{G}^{0} h^{A}\left(v_{\tau}\right) c_{q g \rightarrow q} \int_{0}^{1} d z\left(z-z^{s}\right) \gamma_{q \rightarrow q g}(z) \\
= & -\tilde{G}^{0} h^{A}\left(v_{\tau}\right) c_{q g \rightarrow q} C_{Q}\left\{2 \psi(s+2)+2 \gamma_{E}-\frac{1}{(s+1)(s+2)}-\frac{17}{6}\right\} \\
B_{\left\{\kappa \tilde{Q}^{0}\right\}}^{\prime}(s)= & -\kappa \tilde{Q}^{0} h^{A}\left(v_{\tau}\right) c_{q \bar{q} \rightarrow g} \int_{0}^{1} d z z \gamma_{g \rightarrow q \bar{q}}(z)=-\kappa \tilde{Q}^{0} h^{A}\left(v_{\tau}\right) c_{q \bar{q} \rightarrow g} \frac{1}{6} \\
C_{\left\{\tilde{G}^{0\}}\right.}^{\prime}(s)+n_{f} D_{\left\{\kappa \tilde{Q}^{0\}}\right.}^{\prime}(s)= & -\tilde{G}^{0} h^{A}\left(v_{\tau}\right) c_{g g \rightarrow g} \int_{0}^{1} d z\left(z-z^{s}\right) \gamma_{g \rightarrow g g}(z) \\
& -\kappa \tilde{Q}^{0} h^{A}\left(v_{\tau}\right) c_{g q \rightarrow q} n_{f} \int_{0}^{1} d z z \gamma_{q \rightarrow g q}(z) \\
= & -\tilde{G}^{0} h^{A}\left(v_{\tau}\right) c_{g g \rightarrow g} C_{G}\left\{2 \psi(s+2)+2 \gamma_{E}-4 \frac{s^{2}+3 s+3}{s(s+1)(s+2)(s+3)}\right. \\
& -\frac{11}{6}+\frac{\kappa \tilde{Q}^{0}}{\left.\tilde{G}^{0} \frac{c_{g q \rightarrow q}}{c_{g g \rightarrow g}} \frac{4 C_{Q}}{3 C_{G}} n_{f}\right\}} \\
E_{\left\{\kappa \tilde{Q}^{0\}}\right.}^{\prime}(s)= & -\kappa \tilde{Q}^{0} h^{A}\left(v_{\tau}\right) c_{q \bar{q} \rightarrow g} \int_{0}^{1} d z z^{s} \gamma_{g \rightarrow q \bar{q}}(z) \\
= & -\kappa \tilde{Q}^{0} h^{A}\left(v_{\tau}\right) c_{q \bar{q} \rightarrow g} \frac{1}{2} \frac{s^{2}+3 s+4}{(s+1)(s+2)(s+3)} \cdot
\end{aligned}
$$

The constants $c_{a b \rightarrow c}$ in (51) are given after eqs. (21) and (22). The dimensionless function $h^{A}$ contains the nuclear dependence, eqs. (29) and (30),

$$
\begin{aligned}
h^{A}\left(v_{\tau}\right) & =\frac{9 \pi A^{1 / 3}}{r_{0}^{2}} \frac{v_{\tau} t}{|x| P} \\
& =\frac{9 \pi A^{1 / 3}}{r_{0}^{2} \Lambda^{2}} \exp \left[-\ln \left(\frac{Q_{0}^{2}}{\Lambda^{2}}\right) \exp \left(-2 \pi b v_{\tau} \chi\right)\right] .
\end{aligned}
$$

Finally the scattering kernels are obtained as:

$$
\begin{aligned}
S_{\left\{\kappa \tilde{Q}^{0}\right\}}^{(q q)}(s) & =\frac{1}{4} \alpha_{s}\left(v_{\tau} t\right) \kappa \tilde{Q}^{0} h^{A}\left(v_{\tau}\right) \Sigma_{q q \rightarrow q q} & S_{\left\{\kappa \tilde{Q}^{0}\right\}}^{(q g)}(s) & =\frac{1}{4} \alpha_{s}\left(v_{\tau} t\right) \kappa \tilde{Q}^{0} h^{A}\left(v_{\tau}\right) \Sigma_{q g \rightarrow q g} \\
S_{\left\{\tilde{G}^{0}\right\}}^{(g q)}(s) & =\frac{1}{4} \alpha_{s}\left(v_{\tau} t\right) \tilde{G}^{0} h^{A}\left(v_{\tau}\right) \Sigma_{g q \rightarrow g q} & S_{\left\{\tilde{G}^{0}\right\}}^{(g g)}(s) & =\frac{1}{4} \alpha_{s}\left(v_{\tau} t\right) \tilde{G}^{0} h^{A}\left(v_{\tau}\right) \Sigma_{g g \rightarrow g g} .
\end{aligned}
$$

These scattering kernels result from the operators (23) in the limit of the "small-angle approximation" or "Landau approximation" [30], in which one restricts to scatterings with small transverse momentum exchange $p_{\perp}^{2} \ll \hat{s}<P^{2}$. In this case the scattering operator (23) simplifies considerably to

$$
\begin{aligned}
\hat{S}_{a b}\{\tilde{A}\} B \approx & \int_{0}^{\infty} d \tau_{1} \int_{0}^{1} \frac{d x_{1}}{x_{1}} \int_{-1}^{0} \frac{d x_{2}}{x_{2}} \int_{0}^{\infty} d p_{\perp}^{2} B\left(x_{1}, \tau, t\right) \tilde{A}\left(x_{2}\right) \\
& \times\left.\rho_{N} \frac{d \hat{\sigma}_{a b \rightarrow a b}}{d p_{\perp}^{2}}\right|_{p_{\perp}^{2}=\frac{|x| P}{\tau}} \delta\left(x_{2}^{\prime}-x\right) \delta\left(\tau-\frac{|x| P}{p_{\perp}^{2}}\right) .
\end{aligned}
$$


where scattering at small angles implies [c.f. eq. (24)]

$$
x_{1}=x_{1}^{\prime}+\frac{p_{\perp}^{2}}{\left(x_{1}^{\prime}-x_{2}\right) P^{2}} \approx x_{1}^{\prime} \quad, \quad x_{2}=x_{2}^{\prime}+\frac{p_{\perp}^{2}}{\left(x_{2}^{\prime}-x_{1}\right) P^{2}} \approx x_{2}^{\prime}
$$

and the parton-parton cross-sections $d \hat{\sigma}_{a b \rightarrow a b} / d p_{\perp}^{2}$ given by (26) and (27) can be replaced by their small-angle contributions

$$
\frac{d \hat{\sigma}_{a b \rightarrow a b}}{d p_{\perp}^{2}} \approx \frac{\pi \alpha_{s}^{2}}{p_{\perp}^{4}} \Sigma_{a b \rightarrow a b}
$$

where the constants $\Sigma_{a b \rightarrow a b}$ follow from the squared matrix elements $\left|\overline{\mathcal{M}}_{a b \rightarrow a b}\right|^{2}$, eq. (27), by observing that at small angles $p_{\perp}^{2}=-\hat{t} \ll \hat{s}$ and keeping only the leading terms in $p_{\perp}^{2} / \hat{s}$ :

$$
\begin{aligned}
& \Sigma_{q q \rightarrow q q} \equiv \Sigma_{q_{i} q_{j} \rightarrow q_{i} q_{j}}=\Sigma_{q_{i} \bar{q}_{j} \rightarrow q_{i} \bar{q}_{j}}=\Sigma_{q_{i} q_{i} \rightarrow q_{i} q_{i}}=\Sigma_{\bar{q}_{i} \bar{q}_{i} \rightarrow \bar{q}_{i} \bar{q}_{i}}=\Sigma_{q_{i} \bar{q}_{i} \rightarrow q_{i} \bar{q}_{i}}=\frac{8}{9} \\
& \Sigma_{q g \rightarrow q g} \equiv \Sigma_{q_{i} g \rightarrow q_{i} g}=\Sigma_{\bar{q}_{i} g \rightarrow \bar{q}_{i} g}=2 \\
& \Sigma_{g q \rightarrow g q} \equiv \Sigma_{g q_{i} \rightarrow g q_{i}}=\Sigma_{\bar{g} q_{i} \rightarrow g \bar{q}_{i}}=2 \\
& \Sigma_{g g \rightarrow g g}=\frac{9}{2} .
\end{aligned}
$$

Returning to the eigenvalue equations (48) and combining them, one obtains

$$
\left[\mu+\lambda_{+}(s)\right]\left[\mu+\lambda_{-}(s)\right]=0
$$

which implies

$$
\begin{aligned}
& \lambda_{+}(s)=-\frac{1}{2}\left[F_{q q}(s)+F_{g g}(s)\right]+\frac{1}{2} \sqrt{\left[F_{q q}(s)-F_{g g}(s)\right]^{2}+4 F_{q g}(s) F_{g q}(s)} \\
& \lambda_{-}(s)=-\frac{1}{2}\left[F_{q q}(s)+F_{g g}(s)\right]-\frac{1}{2} \sqrt{\left[F_{q q}(s)-F_{g g}(s)\right]^{2}+4 F_{q g}(s) F_{g q}(s)}
\end{aligned}
$$

and, corresponding to these functions, the constraints

$$
\frac{\mathcal{N}_{g}}{\mathcal{N}_{q}}=\frac{F_{g q}(s)}{F_{g g}(s)+\lambda_{+}(s)}, \quad \text { for } \quad \mu=-\lambda_{+},
$$

or

$$
\frac{\mathcal{N}_{g}}{\mathcal{N}_{q}}=\frac{F_{g q}(s)}{F_{g g}(s)+\lambda_{-}(s)}, \quad \text { for } \quad \mu=-\lambda_{-} .
$$

Thus the solutions are given by 


$$
\begin{aligned}
& \mathcal{Q}(x, t)=\mathcal{N}_{q}\left(\frac{1}{x}\right)^{s} \exp \left[\lambda_{+}(s) \chi(t)\right] \\
& G(x, t)=\frac{\mathcal{N}_{q} F_{g q}(s)}{F_{g g}(s)+\lambda_{+}(s)}\left(\frac{1}{x}\right)^{s} \exp \left[\lambda_{+}(s) \chi(t)\right],
\end{aligned}
$$

or

$$
\begin{aligned}
\mathcal{Q}(x, t) & =\mathcal{N}_{q}\left(\frac{1}{x}\right)^{s} \exp \left[\lambda_{-}(s) \chi(t)\right] \\
G(x, t) & =\frac{\mathcal{N}_{q} F_{g q}(s)}{F_{g g}(s)+\lambda_{-}(s)}\left(\frac{1}{x}\right)^{s} \exp \left[\lambda_{-}(s) \chi(t)\right],
\end{aligned}
$$

or by a linear combination of these two solutions. In general one can write the solutions for the parton number distributions $\wp=\mathcal{Q} / x=\sum_{i}\left(q_{i}+\bar{q}_{i}\right)$ and $g=G / x$ as:

$$
\begin{aligned}
& \wp(x, t) d x=\frac{d x}{x^{s+1}}\left[a_{+} e^{\lambda_{+}(s) \chi(t)}+a_{-} e^{\lambda_{-}(s) \chi(t)}\right] \\
& g(x, t) d x=\frac{d x}{x^{s+1}}\left[\frac{a_{+} F_{g q}}{F_{g g}+\lambda_{+}} e^{\lambda_{+}(s) \chi(t)}+\frac{a_{-} F_{g q}}{F_{g g}+\lambda_{-}} e^{\lambda_{-}(s) \chi(t)}\right] .
\end{aligned}
$$

Fig. 2 shows the behaviour of the functions $F_{q q}, F_{q g}, F_{g q}, F_{g g}$ and Fig. 3 of $\lambda_{+}, \lambda_{-}$, and the derivatives $\lambda_{+}^{\prime}, \lambda_{+}^{\prime \prime}$ with respect to the variable $s$. The three cases a)-c) correspond to different nuclear density of the medium as controlled by the nuclear mass number entering the function $h^{A}$, eq. (52): a) $A=0$ (free space), b) $A=50$ (moderately dense medium), and c) $A=200$ (dense medium). Evidently $\lambda_{+}$vanishes at $s=s_{0} \simeq 1.0 ; 1.1 ; 1.2$ for the cases a); b); c), respectively, and is positive for $s<s_{0}$, and negative for $s>s_{0}$. From (64) it is obvious that the number distributions of shower partons behave as $d x / x^{s+1}$, so that one can interpret the region with $s<s_{0}$ as the developing stage of the shower, with $s=s_{0}$ representing the situation at the shower maximum, and the region with $s>s_{0}$ as the fading stage leading to the tail end of the shower. The variable $s$ therefore indicates the shower age at a given value of $x$ : in a 'young' shower $\left(s<s_{0}\right)$ the number of shower particles increases with age, while in an 'old' shower $\left(s>s_{0}\right)$, it decreases.

Solutions corresponding to a certain initial condition are obtained by summing up these parton shower functions with respect to the variable $s$, weighting them with an appropriate amplitude. This procedure is known as the Mellin transformation method. It has been 
applied extensively to similar problems of electron-photon showers in the early days of cosmic ray theory [31,32]. The reason for introducing Mellin transforms of the parton shower functions is that they are more easily determined than the original functions. Let me denote by $M_{q}\left(M_{g}\right)$ the Mellin transform of the function $\wp(g)$,

$$
M_{q}(s, t)=\int_{0}^{1} d x x^{s} \wp(x, t), \quad M_{g}(s, t)=\int_{0}^{1} d x x^{s} g(x, t)
$$

then the inverse transformation is

$$
\wp(x, t)=\frac{1}{2 \pi i} \int_{c-i \infty}^{c+i \infty} d s \frac{M_{q}(s, t)}{x^{s+1}}, \quad g(x, t)=\frac{1}{2 \pi i} \int_{c-i \infty}^{c+i \infty} d s \frac{M_{g}(s, t)}{x^{s+1}}
$$

where $s$ is now a complex parameter, and the path of the integral is running paralell to the imaginary axis. By analytic continuation of the shower functions (64) to complex values of $s$, one obtains the solutions

$$
\begin{aligned}
& \wp(x, t)=\frac{1}{2 \pi i} \int_{c-i \infty}^{c+i \infty} d s \frac{1}{x^{s+1}}\left[a_{+} e^{\lambda_{+}(s) \chi(t)}+a_{-} e^{\lambda_{-}(s) \chi(t)}\right] \\
& g(x, t)=\frac{1}{2 \pi i} \int_{c-i \infty}^{c+i \infty} d s \frac{1}{x^{s+1}}\left[\frac{a_{+} F_{g q}}{F_{g g}+\lambda_{+}} e^{\lambda_{+}(s) \chi(t)}+\frac{a_{-} F_{g q}}{F_{g g}+\lambda_{-}} e^{\lambda_{-}(s) \chi(t)}\right]
\end{aligned}
$$

where $a_{+}$and $a_{-}$are functions of the complex parameter $s$, and are determined by the initial conditions imposed on $\wp$ and $g$.

I will now discuss the two cases of 1) a parton shower initiated by a quark, and 2) a parton shower triggered by a primary gluon. In accord with the physical picture that was outlined in Sec. 2, the shower axis $z$ is defined along the momentum of the primary quark or gluon, so that the incident parton has zero transverse momentum. Since I consider here the longitudinal evolution only, the time development of the parton cascade proceeds by increasingly populating the region with small values of $x$ as the shower develops along the shower axis which corresponds to growing values of $s$. According to eq. (3), the initiating primary parton carries a longitudinal momentum $p_{z 0}=x_{0} P$ and has a initial time-like virtuality $Q_{0}^{2}$ such that its energy is determined by $E_{0}=\sqrt{\left(x_{0} P\right)^{2}+Q_{0}^{2}}=x_{0} P+Q_{0}^{2} /\left(2 x_{0} P\right)$, where $Q_{0} \ll P$ is assumed. 


\subsubsection{Parton shower initiated by an incident quark}

If one considers a parton shower initiated by a primary quark, then the initial conditions at time $t=t_{0}$ are

$$
\wp\left(x, t_{0}\right)=\delta\left(x_{0}-x\right) \delta\left(E_{0}-P \sqrt{x_{0}^{2}+\frac{Q_{0}^{2}}{P^{2}}}\right), \quad g\left(x, t_{0}\right)=0 .
$$

Inserting this initial condition into (65), one has at $t_{0}=0$,

$$
M_{q}\left(s, t_{0}\right)=x_{0}^{s}, \quad M_{g}\left(s, t_{0}\right)=0,
$$

which means that

$$
\wp(x, t=0)=\frac{1}{2 \pi i} \int_{c-i \infty}^{c+i \infty} d s\left(\frac{x_{0}}{x}\right)^{s} \frac{1}{x}, \quad g(x, t=0)=0 .
$$

The parameters $a_{+}$and $a_{-}$are now completely determined by (68)-(70), together with (67),

$$
a_{+}+a_{-}=x_{0}^{s}, \quad \frac{a_{+} F_{g q}}{F_{g g}+\lambda_{+}}+\frac{a_{-} F_{g q}}{F_{g g}+\lambda_{-}}=0 .
$$

If one introduces functions $H_{+}$and $H_{-}$through

$$
a_{+}=H_{+}(s) x_{0}^{s}, \quad a_{-}=H_{-}(s) x_{0}^{s},
$$

or

$$
H_{+}(s)=\frac{F_{g g}(s)+\lambda_{+}(s)}{\lambda_{+}(s)-\lambda_{-}(s)}, \quad H_{-}(s)=-\frac{F_{g g}(s)+\lambda_{-}(s)}{\lambda_{+}(s)-\lambda_{-}(s)}
$$

one can now express the solutions for the differential spectra of secondary quarks and gluons produced by a primary quark as

$$
\begin{aligned}
& \wp(x, t) d x=\frac{1}{2 \pi i} \frac{d x}{x} \int_{c-i \infty}^{c+i \infty} d s\left(\frac{x_{0}}{x}\right)^{s}\left[H_{+}(s) e^{\lambda_{+}(s) \chi(t)}+H_{-}(s) e^{\lambda_{-}(s) \chi(t)}\right] \\
& g(x, t) d x=\frac{1}{2 \pi i} \frac{d x}{x} \int_{c-i \infty}^{c+i \infty} d s\left(\frac{x_{0}}{x}\right)^{s} \frac{L(s)}{\sqrt{s}}\left[e^{\lambda_{+}(s) \chi(t)}-e^{\lambda_{-}(s) \chi(t)}\right],
\end{aligned}
$$

where

$$
L(s)=\frac{\sqrt{s} F_{g q}(s)}{\lambda_{+}(s)-\lambda_{-}(s)} .
$$


Correspondingly, one can evaluate the integral spectra of secondary quarks and gluons, that is the integrated number densities of partons with longitudinal momentum fractions greater than a given value $x$,

$$
\begin{aligned}
& I_{q}(x, t)=\int_{x}^{x_{0}} d x^{\prime} q\left(x^{\prime}, p_{\perp}^{2}, t\right)=\frac{1}{2 \pi i} \int_{c-i \infty}^{c+i \infty} \frac{d s}{s}\left(\frac{x_{0}}{x}\right)^{s}\left[H_{+}(s) e^{\lambda_{+}(s) \chi(t)}+H_{-}(s) e^{\lambda_{-}(s) \chi(t)}\right] \\
& I_{g}(x, t)=\int_{x}^{x_{0}} d x^{\prime} g\left(x^{\prime}, p_{\perp}^{2}, t\right)=\frac{1}{2 \pi i} \int_{c-i \infty}^{c+i \infty} \frac{d s}{s^{3 / 2}}\left(\frac{x_{0}}{x}\right)^{s} L(s)\left[e^{\lambda_{+}(s) \chi(t)}-e^{\lambda_{-}(s) \chi(t)}\right] .(76)
\end{aligned}
$$

\subsubsection{Parton shower initiated by an incident gluon}

If one considers instead of a primary quark an incident gluon, the initial conditions are

$$
\wp\left(x, t_{0}\right)=0, \quad g\left(x, t_{0}\right)=\delta\left(x_{0}-x\right) \delta\left(E_{0}-P \sqrt{x_{0}^{2}+\frac{Q_{0}^{2}}{P^{2}}}\right),
$$

the procedure is completely analogous. In this case one has instead of (74) for the differential spectra:

$$
\begin{aligned}
& \wp(x, t) d x=\frac{1}{2 \pi i} \frac{d x}{x} \int_{c-i \infty}^{c+i \infty} d s\left(\frac{x_{0}}{x}\right)^{s} \sqrt{s} J(s)\left[e^{\lambda_{+}(s) \chi(t)}-e^{\lambda_{-}(s) \chi(t)}\right] \\
& g(x, t) d x=\frac{1}{2 \pi i} \frac{d x}{x} \int_{c-i \infty}^{c+i \infty} d s\left(\frac{x_{0}}{x}\right)^{s}\left[H_{-}(s) e^{\lambda_{+}(s) \chi(t)}+H_{+}(s) e^{\lambda_{-}(s) \chi(t)}\right]
\end{aligned}
$$

where

$$
J(s)=\frac{1}{\sqrt{s}} \frac{F_{q g}(s)}{\lambda_{+}(s)-\lambda_{-}(s)} .
$$

For the integral spectra one has instead of (76):

$$
\begin{aligned}
& I_{q}(x, t)=\frac{1}{2 \pi i} \int_{c-i \infty}^{c+i \infty} \frac{d s}{\sqrt{s}}\left(\frac{x_{0}}{x}\right)^{s} J(s)\left[e^{\lambda_{+}(s) \chi(t)}-e^{\lambda_{-}(s) \chi(t)}\right] \\
& I_{g}(x, t)=\frac{1}{2 \pi i} \int_{c-i \infty}^{c+i \infty} \frac{d s}{s}\left(\frac{x_{0}}{x}\right)^{s}\left[H_{-}(s) e^{\lambda_{+}(s) \chi(t)}+H_{+}(s) e^{\lambda_{-}(s) \chi(t)}\right] .
\end{aligned}
$$

\subsubsection{Saddle point evaluation of the parton spectra}

The integrals $F=\wp, g, I_{q}, I_{g}$ can be evaluated by numerical computation of the contour integral in the $s$ plane, but alternatively one can also obtain analytical forms by employing the saddle point method which I will outline now. First note that Fig. 3 exhibits the fact that $\lambda_{+}>\lambda_{-}$for all values of $s$, so that one can neglect the second term in these integrals 
$\left(\propto \exp \left[\lambda_{-} \chi\right]\right)$ compared to the first term $\left(\propto \exp \left[\lambda_{+} \chi\right]\right)$. Therefore, dropping the second term the typical structure of the integrals is of the form

$$
\begin{aligned}
F(x, t) & =\frac{1}{2 \pi i} \int_{c-i \infty}^{c+i \infty} d s s^{-k}\left(\frac{x_{0}}{x}\right)^{s} \mathcal{H}(s) e^{\lambda_{+}(s) \chi(t)} \\
& =\frac{1}{2 \pi i} \int_{c-i \infty}^{c+i \infty} d s \mathcal{H}(s) \exp \left[\lambda_{+}(s) \chi+y s-k \ln s\right],
\end{aligned}
$$

where the symbol $\mathcal{H}$ under the integral stands for the functions $H_{+}, H_{-}, L, J$, the variable $y=\ln \left(x_{0} / x\right)$, and the values of the power $k$ are $-1 / 2,0,1 / 2,1$, or $3 / 2$, as can be read off the formulae of Secs. 3.3.1 and 3.3.2. Now, the functions $\mathcal{H}$ are slowly varying with $s$, so that the integrand in (81) is a product of a function $\mathcal{H}$ with a weak $s$-dependence times an exponential of the form $\exp [\phi(s)]$, where

$$
\phi(s)=\lambda_{+}(s) \chi+y s-k \ln s
$$

If $\partial \phi / \partial s=0$ and $\partial^{2} \phi / \partial s^{2}>0$, at some point $s=\bar{s}$, then the integrand in (81) may be approximated by a Gaussian function on the path parallel to the imaginary axis crossing the real axis at $s=\bar{s}$, with the slowly varying functions $\mathcal{H}(s)$ treated as constants and evaluated at $\bar{s}$. Thus, by expanding $\phi(s)$ around $s=\bar{s}$ where $d \phi / d s=0$, one can evaluate the integrals by the saddle point method (see e.g. [31]),

$$
F(x, t)=\left(\left.2 \pi \frac{\partial \phi}{\partial s}\right|_{s=\bar{s}}\right)^{-1 / 2}\left(\frac{x_{0}}{x}\right)^{\bar{s}} \exp \left[\lambda_{+}(\bar{s}) \chi\right]
$$

with the saddle point $\bar{s}$ defined by the equation

$$
\frac{\partial \phi}{\partial s}=\lambda_{+}^{\prime}(\bar{s}) t+y-\frac{k}{\bar{s}}=0
$$

which establishes a correlation between $y=\ln \left(x_{0} / x\right)$ and $\chi$, i.e. between the momentum distribution and the time evolution variable $\chi$. Applying this formalism, one obtains the following results for the parton spectra $F=\wp, g, I_{q}, I_{g}$ (dropping the bar on the $s$, i.e. $s \equiv \bar{s}$ is the saddle point):

1) For a primary quark of longitudinal momentum fraction $x_{0}$, 


$$
\begin{aligned}
& \wp(x, t) d x=\frac{1}{\sqrt{2 \pi}} \frac{H_{+}(s)}{\sqrt{\lambda_{+}^{\prime \prime}(s) \chi}}\left(\frac{x_{0}}{x}\right)^{s} \frac{d x}{x} e^{\lambda_{+}(s) \chi}, \quad \chi(t)=-\frac{1}{\lambda_{+}^{\prime}(s)} \ln \left(\frac{x_{0}}{x}\right) \\
& g(x, t) d x=\frac{1}{\sqrt{2 \pi s}} \frac{L(s)}{\sqrt{\lambda_{+}^{\prime \prime}(s) \chi+1 /\left(2 s^{2}\right)}}\left(\frac{x_{0}}{x}\right)^{s} \frac{d x}{x} e^{\lambda_{+}(s) \chi}, \quad \chi(t)=-\frac{1}{\lambda_{+}^{\prime}(s)}\left[\ln \left(\frac{x_{0}}{x}\right)-\frac{1}{2 s}\right] \\
& I_{q}(x, t)=\frac{1}{\sqrt{2 \pi s^{2}}} \frac{H_{+}(s)}{\sqrt{\lambda_{+}^{\prime \prime}(s) \chi+1 / s^{2}}}\left(\frac{x_{0}}{x}\right)^{s} e^{\lambda_{+}(s) \chi}, \quad \chi(t)=-\frac{1}{\lambda_{+}^{\prime}(s)}\left[\ln \left(\frac{x_{0}}{x}\right)-\frac{1}{s}\right] \\
& I_{g}(x, t)=\frac{1}{\sqrt{2 \pi s^{3}}} \frac{L(s)}{\sqrt{\lambda_{+}^{\prime \prime}(s) \chi+3 /\left(2 s^{2}\right)}}\left(\frac{x_{0}}{x}\right)^{s} e^{\lambda_{+}(s) \chi}, \quad \chi(t)=-\frac{1}{\lambda_{+}^{\prime}(s)}\left[\ln \left(\frac{x_{0}}{x}\right)-\frac{3}{2 s}\right] .
\end{aligned}
$$

2) For a primary gluon of longitudinal momentum fraction $x_{0}$,

$$
\begin{aligned}
& \wp(x, t) d x=\sqrt{\frac{s}{2 \pi}} \frac{J(s)}{\sqrt{\lambda_{+}^{\prime \prime}(s) \chi-1 /\left(2 s^{2}\right)}}\left(\frac{x_{0}}{x}\right)^{s} \frac{d x}{x} e^{\lambda_{+}(s) \chi}, \quad \chi(t)=-\frac{1}{\lambda_{+}^{\prime}(s)}\left[\ln \left(\frac{x_{0}}{x}\right)+\frac{1}{2 s}\right] \\
& g(x, t) d x=\frac{1}{\sqrt{2 \pi}} \frac{H_{-}(s)}{\sqrt{\lambda_{+}^{\prime \prime}(s) \chi}}\left(\frac{x_{0}}{x}\right)^{s} \frac{d x}{x} e^{\lambda_{+}(s) \chi}, \quad \chi(t)=-\frac{1}{\lambda_{+}^{\prime}(s)} \ln \left(\frac{x_{0}}{x}\right) \\
& I_{q}(x, t)=\frac{1}{\sqrt{2 \pi s}} \frac{J(s)}{\sqrt{\lambda_{+}^{\prime \prime}(s) \chi+1 /\left(2 s^{2}\right)}}\left(\frac{x_{0}}{x}\right)^{s} e^{\lambda_{+}(s) \chi}, \quad \chi(t)=-\frac{1}{\lambda_{+}^{\prime}(s)}\left[\ln \left(\frac{x_{0}}{x}\right)-\frac{1}{2 s}\right] \\
& I_{g}(x, t)=\frac{1}{\sqrt{2 \pi s^{2}}} \frac{H_{-}(s)}{\sqrt{\lambda_{+}^{\prime \prime}(s) \chi+1 / s^{2}}}\left(\frac{x_{0}}{x}\right)^{s} e^{\lambda_{+}(s) \chi}, \quad \chi(t)=-\frac{1}{\lambda_{+}^{\prime}(s)}\left[\ln \left(\frac{x_{0}}{x}\right)-\frac{1}{s}\right] .
\end{aligned}
$$

Recall that the time evolution variable $\chi(t)$, defined in (45), relates these spectra to real time $t$ via eq. (46), so that $t$ can be resubstituted if desired, provided $v_{\tau}$ in (42) is known. I would like to remind the reader that one of the advantages for using $\chi$, eq. (45), as evolution variable rather than the laboratory time $t$, was to absorb the nuclear medium dependence and thereby obtain a relatively model independent description. Thus, notwithstanding the uncertainty of medium effects, the properties of the parton shower evolution are essentially controlled by perturbative QCD, but the connection to real time resides in the relation (46). From this point of view, the parton spectra (85) and (86) represent the elementary solutions to the evolution equation (43) under the approximations made in Sec. 3.1 and summarize the longitudinal parton cascade evolution in nuclear matter within the present framework. It is evident that the variation of the number of shower particles with time follows an exponential law with exponent $\lambda_{+}(s) \chi(t)$ with the (implicitely medium dependent) 'absorption coefficient' $\left(-\lambda_{+}\right)$describing the variation with time and the power $(-s)$ controlling the momentum distribution in $x_{0} / x$. 
It is worth noting that the distributions $\wp$ and $g$ reduce to the well known approximate solutions to the $Q^{2}$-evolution according to the DGLAP equations [18], if one sets the nuclear matter density equal to zero. Then $\tau=v_{\tau} t=t$ or $v_{\tau}=1$, because in the absence of scattering and fusion processes the particles can only evolve by successive branchings with the age $\tau$ being equal to laboratory time $t$. The connection between time- and $Q^{2}$-evolution, as discussed in Sec. 2.1, is given by the average relation (9), $Q^{2}=x P / t$. Then, considering the evolution of the gluon distribution alone, at small $x$ the behavior is controlled by the branching kernel $F_{g g}(s)=C(s)$ near $s=0$ [c.f. eq. (50)]. Hence, taking only the dominant term in $F_{g g}$ into account, one has

$$
\frac{\partial}{\partial t} G=-F_{g g} G, \quad F_{g g}(s) \approx-\frac{2 C_{G}}{s}=-\lambda_{+}(s)
$$

where $C_{G}=n_{c}$ as before. Then the saddle point evaluation yields (setting $x_{0}=1$ ):

$$
g(x, t) d x=\frac{1}{2 \pi} \frac{\left[\pi \frac{n_{c}}{b} \ln \left(\frac{\alpha_{s}\left(Q^{2}\right)}{\alpha_{s}\left(Q_{0}^{2}\right)}\right) \ln (1 / x)\right]^{1 / 4}}{\ln (1 / x)} \exp \left[\sqrt{\frac{4}{\pi} \frac{n_{c}}{b} \ln \left(\frac{\alpha_{s}\left(Q^{2}\right)}{\alpha_{s}\left(Q_{0}^{2}\right)}\right) \ln (1 / x)}\right] \frac{d x}{x},
$$

where the time variable $\chi$, when re-expressed in terms of $Q^{2}$ rather than $t$, is given by

$$
\chi\left(Q^{2}\right)=\frac{1}{2 \pi b} \ln \left(\frac{\alpha_{s}\left(Q^{2}\right)}{\alpha_{s}\left(Q_{0}^{2}\right)}\right)=\frac{1}{2 \pi b} \ln \left(\frac{\ln \left(Q_{0}^{2} / \Lambda^{2}\right)}{\ln \left(Q^{2} / \Lambda^{2}\right)}\right),
$$

with $Q_{0}^{2}=P / t_{0}$ and $Q^{2}=x P / t\left(t>t_{0}\right)$. Eq. (88) together with (89) coincides with leading logarithmic behavior of the gluon distribution within the LLA [18].

Let me return now to the parton spectra (85) and (86), and examplarily exhibit some characteristic features of the longitudinal parton shower evolution in nuclear matter, as reflected by the $\chi, x$ and nuclear $(A)$ dependence of the spectra. In what follows I chose the virtuality of the cascade initiating parton $Q_{0}=30 \mathrm{GeV}$. An increase (decrease) of $Q_{0}$ generally results in a larger (smaller) overall multiplicity of secondaries and also shifts the evolution toward earlier (later) times. The initial longitudinal momentum fraction was set to $x_{0}=1$, since the shower development depends only on the ratio $x_{0} / x$. Finally the 
QCD scale $\Lambda$ that enters the running coupling $\alpha_{s}$ was taken equal to $230 \mathrm{MeV}$. In order to exhibit the nuclear medium influence on the shower development, as controlled by eq. (52), I considered as before the three cases: a) $A=0$ (absence of medium), b) $A=50$ (moderately dense medium), and, c) $A=200$ (dense medium).

In Figs. 4 and 5 the parton number densities $\wp(x, t)$ and $g(x, t)$ from (85) and (86), respectively, are plotted for $x=10^{-1}$ and $x=10^{-3}$ versus $\chi$ (it is helpful to keep in mind that for slowly varying $\alpha_{s}$, one has approximately $\chi \propto \ln t$ ). The essential observations are basically the same for both quark and gluon initiated showers:

(i) For a given value of the ratio $x$, the change with "time" $\chi$ of the spectra $\wp$ and $g$ reflects the aging of the shower. The multiplicity initially increases (young shower), goes through a peak (shower maximum), and then decreases again (old shower). The time it takes for a parton shower to evolve from $x_{0}=1$ downwards to some value $x$ naturally increases with decreasing $x$ and at the same time the average multiplicity of produced particles grows rapidly. (The integral spectra $I_{q}$ and $I_{g}$, which are not shown here, exhibit an analogous behaviour.)

(ii) Comparing the cases a) to c), the influence of the nuclear medium manifests itself in a slowing down of the shower development, particularly at later times $(\chi \gtrsim 2)$. In other words, the aging of the cascading partons is more delayed, the denser the medium is. Related to that is the clear increase of the parton multiplicity at a given $x$ with larger nuclear density, because repeated energy-momentum transfers by scatterings and fusions rejuvenate the shower partons and amplify the bremsstrahlung processes.

In order to exhibit the $x$-dependence of the parton spectra at fixed value of $\chi$ let me define the so-called normal spectra 31] at the shower maximum. The normal spectra correspond to an optimum value $\chi=\chi_{0}$, which coincides with the value of $\chi$ that makes the function $\exp [\phi(s)]$ of eq. (82) a maximum, because the other terms change slowly with $\chi$. Thus, $\chi_{0}$ is defined by the equation

$$
\frac{\partial}{\partial \chi} \exp [\phi(s)]=0 \quad \text { at } \chi=\chi_{0}
$$


or

$$
\lambda_{+}(s)+\left(y+\lambda_{+}^{\prime}(s) \chi-\frac{k}{s}\right)\left(\frac{\partial s}{\partial t}\right)_{\chi=\chi_{0}}=0 .
$$

Recalling that eq. (84) is satisfied, one has

$$
\lambda_{+}(s)=0 \quad \text { at } \chi=\chi_{0} .
$$

Hence, at $s=s_{0}$,

$$
\chi_{0}=-\frac{y-k}{\lambda_{+}^{\prime}\left(s_{0}\right)}=-\frac{1}{\lambda_{+}^{\prime}\left(s_{0}\right)}\left[\ln \left(\frac{x_{0}}{x}\right)-k\right] .
$$

The normal spectra of $\wp, g, I_{q}, I_{g}$ and the corresponding optimum values $\chi_{0}$ are easily obtained from eqs. (85) and (86) by putting $s=s_{0}, \lambda_{+}=0$, and $\chi=\chi_{0}$. The values of $s_{0}$, given by $\lambda_{+}\left(s_{0}\right)=0$, and of $\lambda_{+}^{\prime}\left(s_{0}\right)$ can be read off Fig. 2 .

The top part of Fig. 6 displays the typical shape of the normal spectrum around shower maximum, corresponding to $s=s_{0}$ and $\chi=\chi_{0}$. It is interesting that, when plotted as a normalized probability distribution $p\left(\chi_{0}\right)$, this normal spectrum is unique: first, it is the same for quarks and gluons, second, it is independent of the type of primary particle, and, third, it does not depend on the nuclear medium density. On the other hand, the corresponding optimum values $\chi_{0}$ vary significantly for the different cases, as is evident from the lower part of Fig. 6, which shows $\chi_{0}$ versus $x$ for the three aforementioned cases a) $A=0$, b) $A=50$ and c) $A=200$. This behaviour is to be expected, since $\chi_{0}$ marks the characteristic time that it takes for the parton shower to build up the normal spectrum. Looking at Fig. 6, one can conclude:

(i) With decreasing $x$ the values of $\chi_{0}$ increase, roughly as $\ln (1 / x)$. The slope is the same for both quarks and gluons, but $\chi_{0}^{g}$ is always smaller than $\chi_{0}^{q}$, reflecting the fact that the gluons evolve faster due to their larger interaction and radiation probability.

(ii) The effect of the nuclear medium is again a substantial dilation of the shower development, and thus toward the establishment of the normal spectrum. This is clearly reflected by the steepening of the slope of the time scale $\chi_{0}$ when proceeding from the free space case a) to the dense matter case c). 


\section{SUMMARY}

In this paper I presented an analytical method to solve the QCD evolution equations derived in Ref. [1] which describe the cascading of quarks and gluons in a nuclear environment. These evolution equations extend the well known DGLAP equations for jet development in hard QCD processes, to the time evolution of parton cascades in a nuclear medium, including in addition to spontaneous branchings also fusion, scattering and stimulated branching processes. In order to obtain tractable closed solutions to the evolution equations, a number of idealizing approximations were necessary. Therefore, at this point, this approach does of course not provide quantitative predictions of parton transport phenomena in a dense matter environment. The main uncertainty is here the lack of detailed knowledge of the form of the medium influence on the cascade evolution. Since frequency and type of interactions of the partons with the medium must depend crucially on the nuclear density and structure, one must supply some external input. In order to minimize the amount of phenomenological input, the medium influence was therefore hidden into a time evolution variable $\chi(t)$, the relation of which with the laboratory time $t$ however is determined by the specific properties of the nuclear medium. As advantagous trade-off one gains that the calculated properties and spectra of the cascading partons are a rather firm consequence of perturbative QCD proceses in a nuclear medium being characterized by a specific time variable $\chi$.

Of prime interest in this work was the multiplicative behaviour of cascading partons at small $x$, since such soft particles each take away only a negligable portion of the total energy, but form at the same time the bulk of multiplicity. The characteristic properties of parton shower evolution inside nuclear matter with respect to the variables time $(\chi)$, longitudinal momentum fraction $(x)$ and nuclear density $(A)$ can be summarized as follows:

(1) With respect to the rate of time change at fixed value of $x$, a typical parton shower can be divided roughly in three stages: (i) young shower: during the early stage the shower particles rapidly multiply due to branching processes (mainly gluon emission);

(ii) shower maximum: for a short time the number of particles in the cascade remains 
almost constant; (iii) old shower: the increasing dissipation of energy due to branchings as well as a growing number of fusion and scattering processes reduce the number of shower particles at given $x$ during later times.

(2) The mean of the $x$-distribution of shower partons shifts with progressing time to smaller values of $x$, but this development is significantly delayed in a dense matter environment as compared to free space. The $x$-spectra exhibit a unique form around the shower maximum, independent of type of parton and of the nuclear density. This normal spectrum is established after a certain optimum time $\chi=\chi_{0}$, which measures the characteristic time scale that depends on the density of the surrounding medium: the denser the matter, the longer it takes for the partons to build up to their normal spectrum. Also, gluons always reach this point earlier than quarks, because of their larger cross-section and radiation probability.

As said before, the approach presented here has no truly predictive relevance for experimental observables yet. Rather than that it may be a first step towards a more fundamental understanding of "QCD in medium", using well developed methods of renormalization group improved perturbation theory for "QCD in vacuum". I believe that the most important issues to be addressed in the near future within such a program are the following:

(1) The rigorous distinction between the shower particles and the nuclear partons needs to be dropped. Instead both should be treated on the same footing in order to describe a self-consistent dynamical multi-parton system. This would then incorporate also timeand $x$-dependent nuclear parton distributions that receive modifications due to the response of the shower evolution. An interesting aspect of such a dynamical interplay between scatterings, multiplication and recombination of partons is the question of a possible saturation [33] of the parton number densities that may render a finite parton density as $x \rightarrow 0$.

(2) In the context of nuclear structure, the relevance and the form of the age distribution $\mathcal{A}$ 
of vitual shower partons needs to be studied, because it provides an interface between the nuclear medium and the response of the cascading partons to this environment. Therefore $\mathcal{A}$ may be experimentally accessible by comparing inclusive quantities associated with parton evolution in e.g. deep inelastic scattering experiments on hadrons versus heavy nuclei. This issue is of great interest from both theoretical as well as experimental side.

(3) Interference effects which are important particularly in the small $x$-domain need to be taken into account systematically. These include two well known phenomena, namely, a) the coherence of successive soft gluon emissions in the parton cascade leading to destructive interference and a suppression of gluon radiation, and, b) the LandauPomerantchuk-Migdal effect, i.e. the interference of induced gluon emission amplitudes in multiple parton collisions, which also causes a depletion in the gluon radiation spectrum.

(4) A possible extension to include the time development of the transverse momentum distribution of partons as well as the lateral spread of shower particles would be desirable. This would then provide a solution to the cascade development in full four dimensional momentum space, in virtuality $Q^{2}$, longitudinal and transverse momentum components $x$ and $k_{\perp}^{2}$, respectively.

(5) Parallel to the previous items a comparison with a full six-dimensional phase-space analysis, as e.g. possible with Monte Carlo simulations [3], is of great importance to test the accuracy of the analytical solutions. Such a twofold investigation can be explorative for both analytical and numerical solutions of the evolution equations, in the sense that mutual feedback may lead to an improved understanding of the microscopic parton dynamics in nuclear matter. 


\section{ACKNOWLEDGEMENTS}

I would like to thank H.-T. Elze for many inspiring conversations during the course of this work. 


\section{REFERENCES}

[1] K. Geiger and B. Müller, QCD evolution equations for high energy partons in nuclear matter, preprint CERN-TH. 7205/94 (to appear in Phys. Rev. D).

[2] See e.g.: A. H. Mueller (ed), Perturbative Quantum Chromodynamics, Advanced Series on Directions in High Energy Physics, Vol. 5 (World Scientific, Singapore 1989)

[3] K. Geiger and B. Müller, Nucl. Phys. B369, 600 (1992); K. Geiger, Phys. Rev. D 47, $133(1993)$.

[4] K. Geiger and J. I. Kapusta, Phys. Rev. Lett. 70, 1290 (1993). K. Geiger, Phys. Rev. Lett. 71, 3075 (1993); K. Geiger, Phys. Rev. D 48, 4129 (1993).

[5] X. N. Wang and M. Gyulassy, Phys. Rev. D44, 3501 (1991).

[6] P. Aurenche et al., Phys. Rev. D45, 92 (1992).

[7] For a recent overview on the status of high energy nucleus-nucleus collisions, see: Quark Matter '93, Proceedings of the Tenth International Conference on Ultra-relativistic Nucleus-Nucleus Collisions, Borlänge, Sweden, 1993, edited by H. A. Gustafsson et al. [Nucl. Phys. A566, 1c (1994)].

[8] B. Müller, Physics of the Quark-Gluon Plasma, Particle Production in Highly Excited Matter, edited by H. Gutbrod and J. Rafelski (Plenum Press, New York, 1993) p. 11; Quark Gluon Plasma, edited by R. C. Hwa (World Scientific, Singapore 1990).

[9] Proceedings of the Workshop on Pre-equilibrium Parton Dynamics in Heavy Ion Collisions, edited by X. N. Wang, LBL-Report 34831, 1993.

[10] L. Frankfurt and M. Strikman, Phys. Rep. 160, 235 (1988).

[11] N. Karzeev and H. Satz, The quantum mechanical origin of nuclear shadowing, CERNTH. 7115/93 (unpublished).

[12] X.-N. Wang and M. Gyulassy, Phys. Rev. Lett. 68, 1480 (1992); M. Gyulassy and X.-N. 
Wang, Multiple Collisions and Induced Gluon Radiation in QCD, preprint LBL-32682 UC-413, 1993 (unpublished).

[13] Yu. L. Dokshitzer, Sov. Phys. JETP 46, 641 (1977).

[14] G. Altarelli and G. Parisi Nucl. Phys. B126, 298 (1977).

[15] See e.g.: Yu. L. Dokshitzer, V. A. Khoze, A. H. Müller, and S. I. Troyan, Basics of Perturbative QCD, Advanced Series on Directions in High Energy Physics, Vol. 5 (Editions Frontieres, Gif-sur-Yvette Cedex, France, 1991)

[16] V. N. Gribov and L. N. Lipatov, Yad. Fiz. 15, 781 (1972) [Sov. J. Nucl. Phys. 15, 438 (1972)]; 15, 1218 (1972) [15, 675 (1972)]; L. N. Lipatov, ibid. 20, 181 (1974) [20, 94 (1975)].

[17] J. Kogut and L. Susskind, Phys. Rev. D 9, 697 (1974); 9, 3391 (1974).

[18] Yu. L. Dokshitzer, D. I. Dyakonov, and S. I. Troyan, Phys. Rep. 58, 269 (1980).

[19] G. Reya, Phys. Rep. 69, 195 (1981); A. H. Mueller, Phys. Rep. 73, 237 (1981); A. Bassetto, M. Ciafaloni and G. Marchesini, Phys. Rep. 100, 203 (1983).

[20] See e.g.: R. Balescu, Statistical Mechanics of Charged Particles (Interscience Publishers, London 1963); S. de Groot, W. A. van Leuwen and C. G. van Weert, Relativistic Kinetic Theory (North Holland, Amsterdam 1980).

[21] J. C. Collins, D. E. Soper and G. Sterman, in Ref. [2], p. 1.

[22] L. McLerran and R. Venugopalan, preprint TPI-MINN-93-52-T (hep-ph/9311205); preprint TPI-MINN-93-44-T (hep-ph/9309289).

[23] K. Geiger, Nucl. Phys. A 566, 257c (1994).

[24] K. Geiger, Probing the space-time structure of quark and gluon transport in protonnucleus collisions at collider energies, preprint NSF-ITP-93-134 (1993) (submitted to 
Phys. Rev. C).

[25] F. E. Close, J. Qiu and R. G. Roberts, Phys. Rev. D 40, 2820 (1989).

[26] R. D. Fields, Applications of Perturbative QCD, Frontiers in Physics 77, (AddisonWesley, 1989).

[27] B. L. Combridge, Nucl. Phys. B151, 429 (1979); B. Svetitsky, Phys. Rev. D37, 2484 (1988).

[28] There is recent experimental evidence from HERA data that at very small $x \lesssim 10^{-3}$ the gluon structure function of a proton exhibits a "Lipatov enhancement" $x \hat{g}(x) \propto x^{-C}$ with $c \approx 1 / 2$. Nevertheless, for reasons of simplicity mentioned in the text, I will use the approximation of a constant behaviour at small $x$, which corresponds to $C=0$.

[29] A. H. Mueller and J. Qiu, Nucl. Phys. B286, 427 (1986).

[30] A detailed review on small-angle multiple scattering theory can be found in: W. T. Scott, Rev. Mod. Phys. 35, 231 (1963).

[31] B. Rossi, High Energy Particles (Prentice Hall Inc., New York 1952).

[32] J. Nishimura, in Handbuch der Physik, Vol. 96/2, pp. 1 (Springer, Heidelberg 1967).

[33] J. P. Blaizot and A. H. Mueller, Nucl. Phys. B289, 847 (1987); E. M. Levin and M. G. Ryskin, Phys. Rep. 189, 267 (1990). 


\section{FIGURE CAPTIONS}

Figure 1: Diagrammatic representation of the coupled evolution equations (13)-(15) for a) quarks (and similarly antiquarks) and b) gluons. In contrast to the branching processes, the fusion and scattering processes involve interactions of a cascade parton with a nuclear parton (marked by thick lines). In a) the various diagrams correspond to the integral operators $-\hat{A} Q_{i}, \hat{B} G,-\hat{A}^{\prime} Q_{i},-\hat{B}^{\prime} Q_{i}, \sum_{j}\left[\hat{S}_{q q} Q_{j}+\hat{S}_{q \bar{q}} \bar{Q}_{j}\right], \hat{S}_{q g} G$, and in b) to $-\hat{C} G,-\hat{D} G$, $\sum_{j}\left[\hat{E} Q_{j}+\hat{E} \bar{Q}_{j}\right],-\hat{C}^{\prime} G,-\hat{D}^{\prime} G, \sum_{j}\left[\hat{E}^{\prime} Q_{j}+\hat{E}^{\prime} \bar{Q}_{j}\right], \sum_{j}\left[\hat{S}_{g q} Q_{j}+\hat{S}_{g \bar{q}} \bar{Q}_{j}\right], \hat{S}_{g g} G$.

Figure 2: Form of the functions $F_{q q}, F_{q g}, F_{g q}, F_{g g}$, eq. (49), with respect to the variable s. The three plots a)-c) correspond to different nuclear density of the medium measured in terms of nuclear mass number: $A=0$ (free space), b) $A=50$ (moderately dense medium), and c) $A=200$ (dense medium).

Figure 3: Functional behavior of $\lambda_{+}, \lambda_{-}$, eq. (59), and of the derivatives $\lambda_{+}^{\prime}, \lambda_{+}^{\prime \prime}$ versus the variable $s$. The cases a)-c) correspond to Fig. 2.

Figure 4: Primary quark: Time-evolution of the differential spectra for the parton number densities $\wp(x, t)$ and $g(x, t)$ with respect to the variable $\chi(t)$, according to (85), at fixed values $x=10^{-1}$ and $x=10^{-3}$. The virtuality of the cascade initiating quark was chosen $Q_{0}=30 \mathrm{GeV}$. Cases a)-c) as in Fig. 2 .

Figure 5: Primary gluon: Development with time $\chi(t)$ of the parton number densities $\wp(x, t)$ and $g(x, t)$ for the case of a shower initiating gluon, eq. (86), with initial virtuality $Q_{0}=30 \mathrm{GeV}$ and at fixed values $x=10^{-1}$ and $x=10^{-3}$. Cases a)-c) as in Fig. 2.

Figure 6: Top: Unique shape of the normal spectrum around shower maximum in terms of the normalized probability distribution $p\left(\chi_{0}\right)=a\left(x, \chi_{0}\right) / \int d x a\left(x, \chi_{0}\right)$ where $a=\wp, g$. Middle and bottom: The optimum values $\chi_{0}$ for gluons and quarks versus $x$, corresponding to the point of time $\chi(t)$ when the normal spectrum at a certain value of $x$ is established. The three sets of curves a) and c) refer to different nuclear density, as in Fig. 2. The virtuality of the primary quark, respectively gluon, was chosen $Q_{0}=30 \mathrm{GeV}$. 

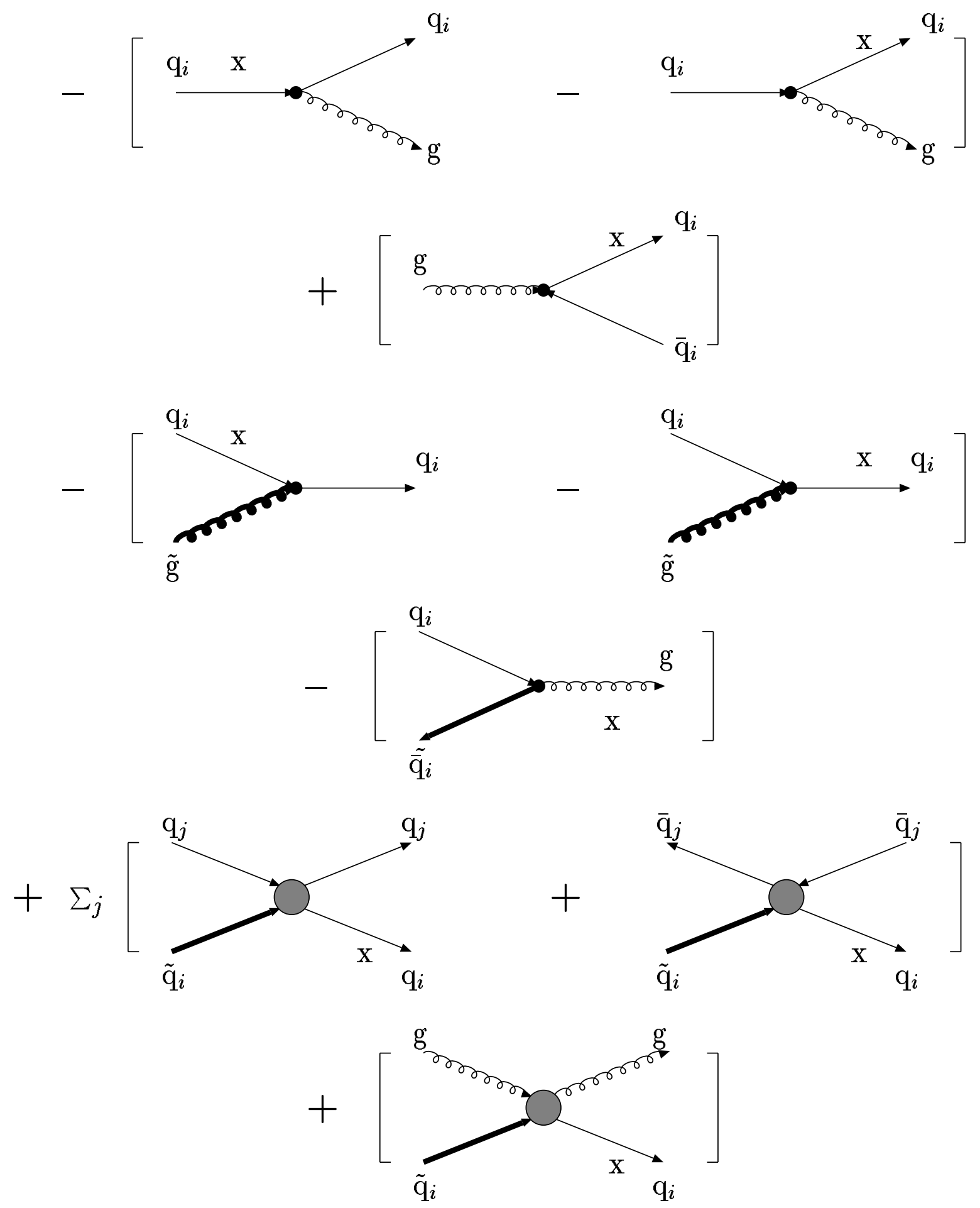

Fig. 1 a) 


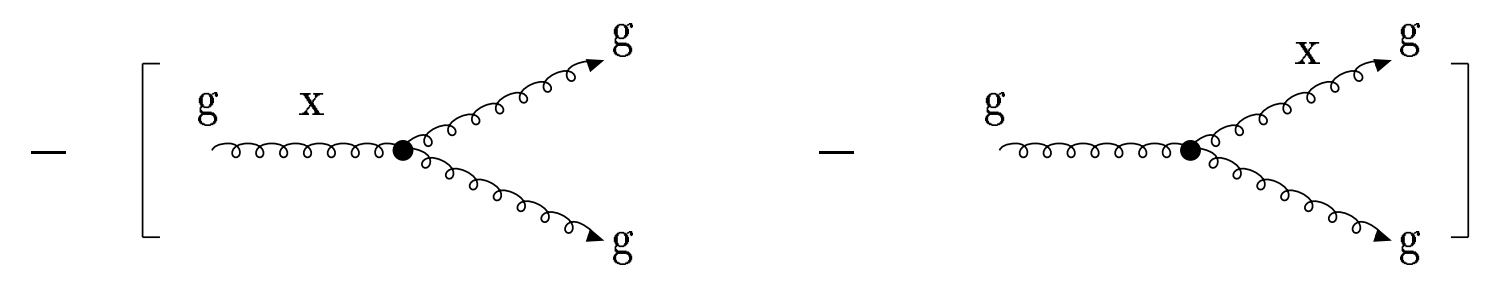

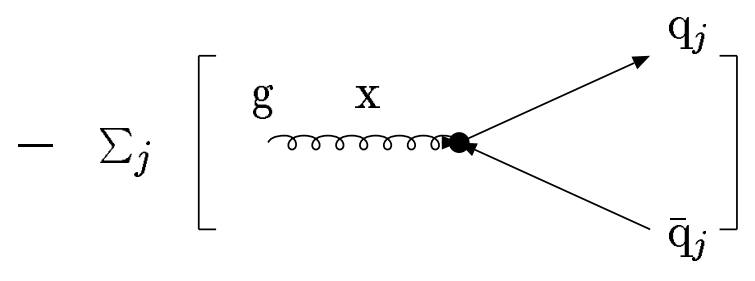
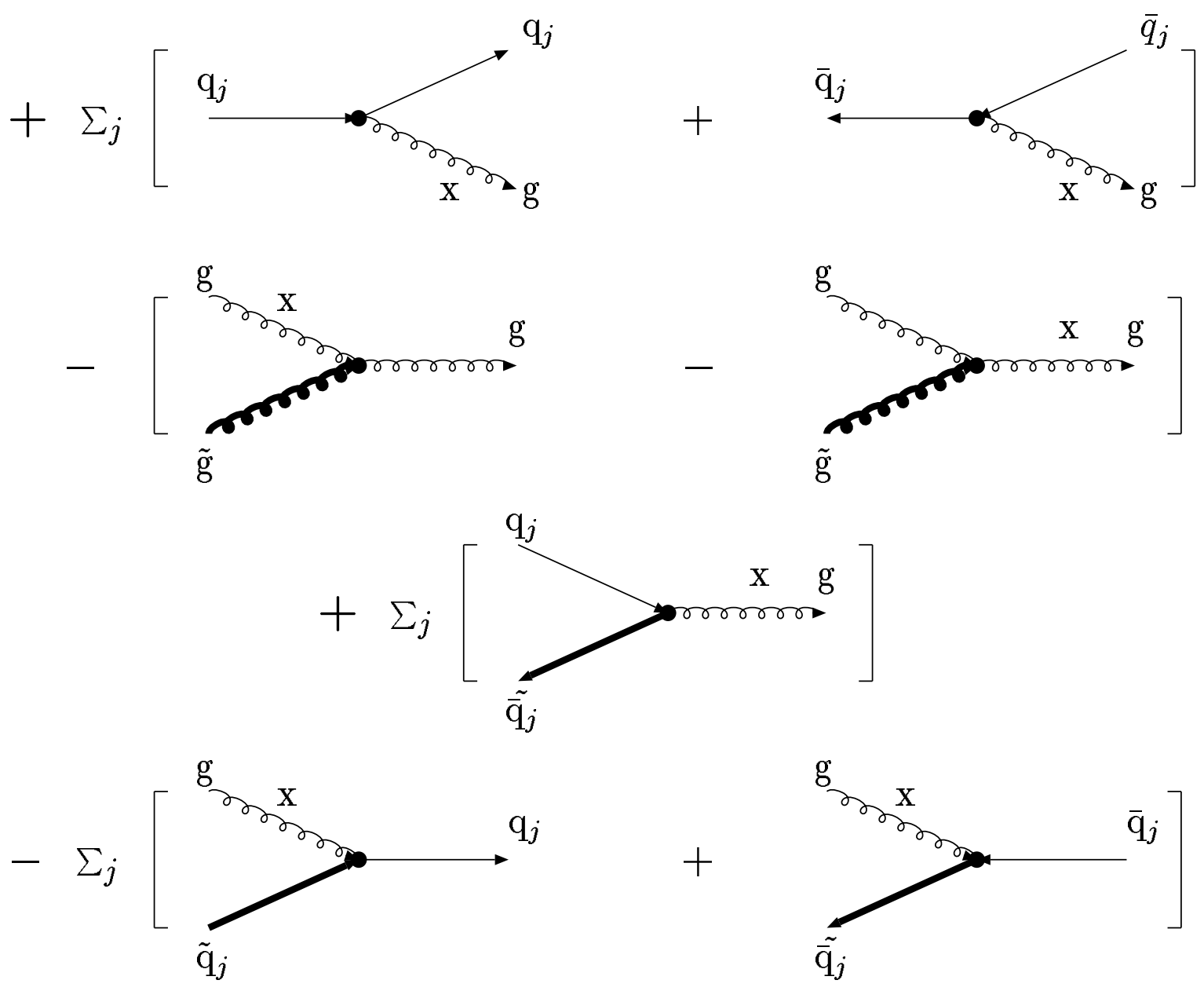

Fig. 1 b) 


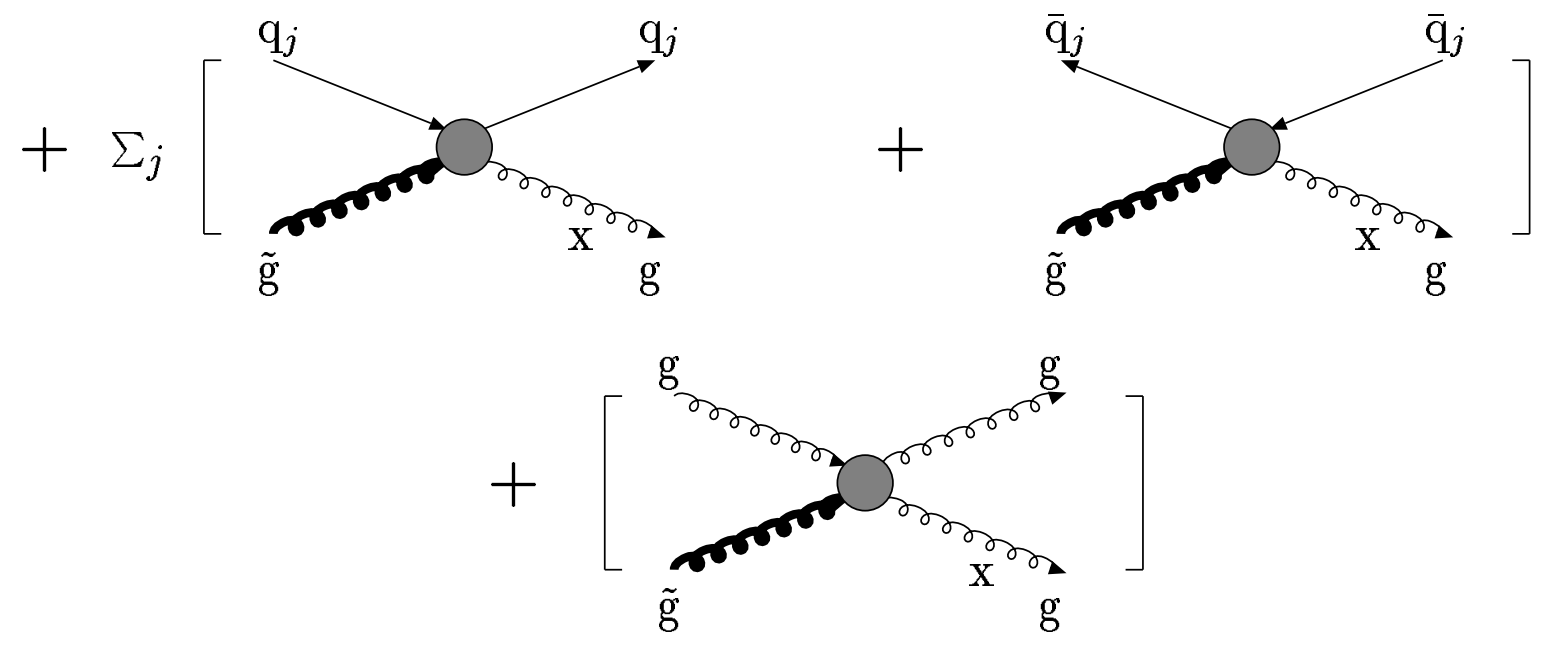

Fig. 1 b) cont'd 
This figure "fig1-1.png" is available in "png" format from: http://arxiv.org/ps/hep-ph/9403409v1 
This figure "fig2-1.png" is available in "png" format from: http://arxiv.org/ps/hep-ph/9403409v1 
This figure "fig3-1.png" is available in "png" format from: http://arxiv.org/ps/hep-ph/9403409v1 


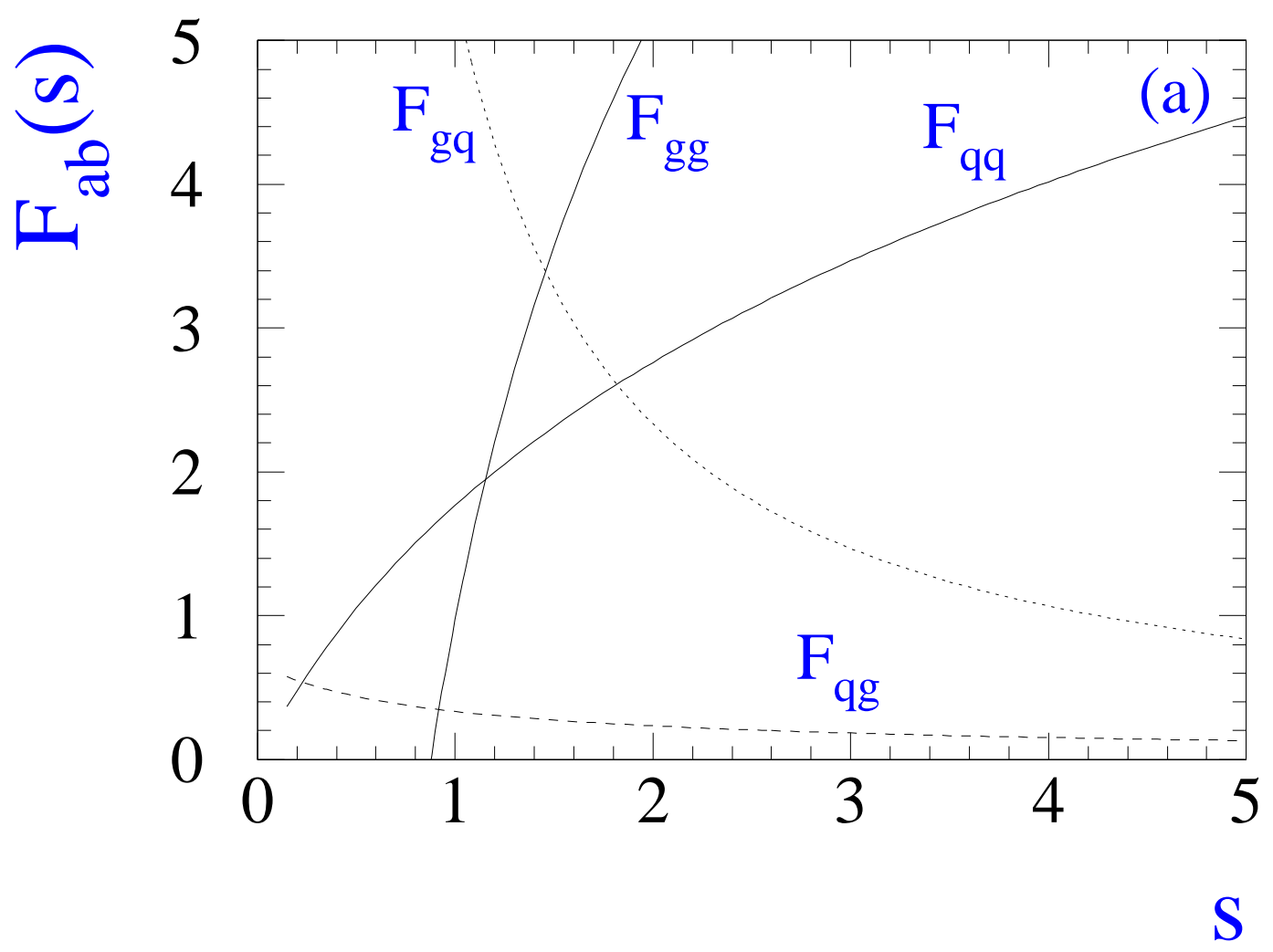




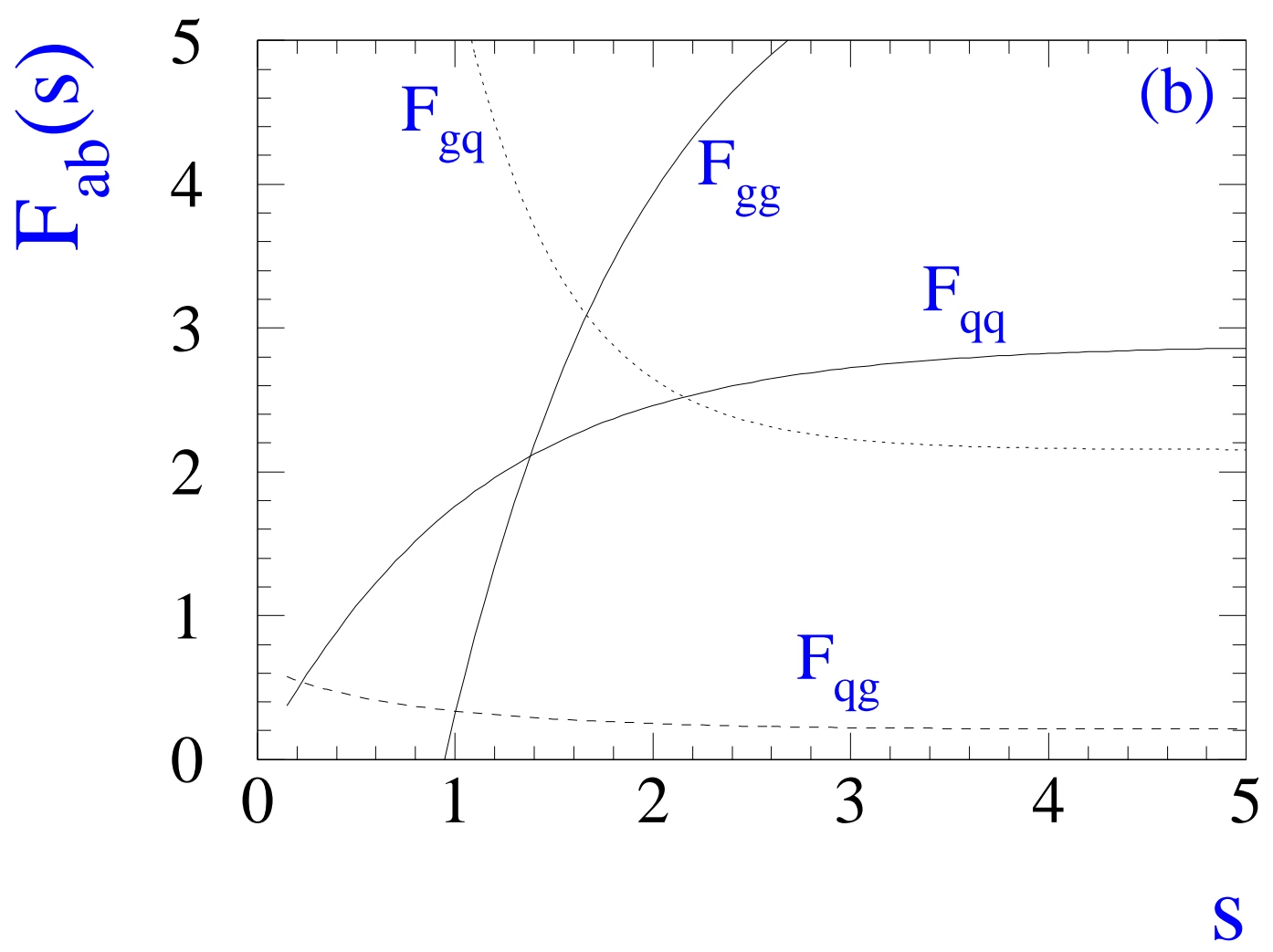




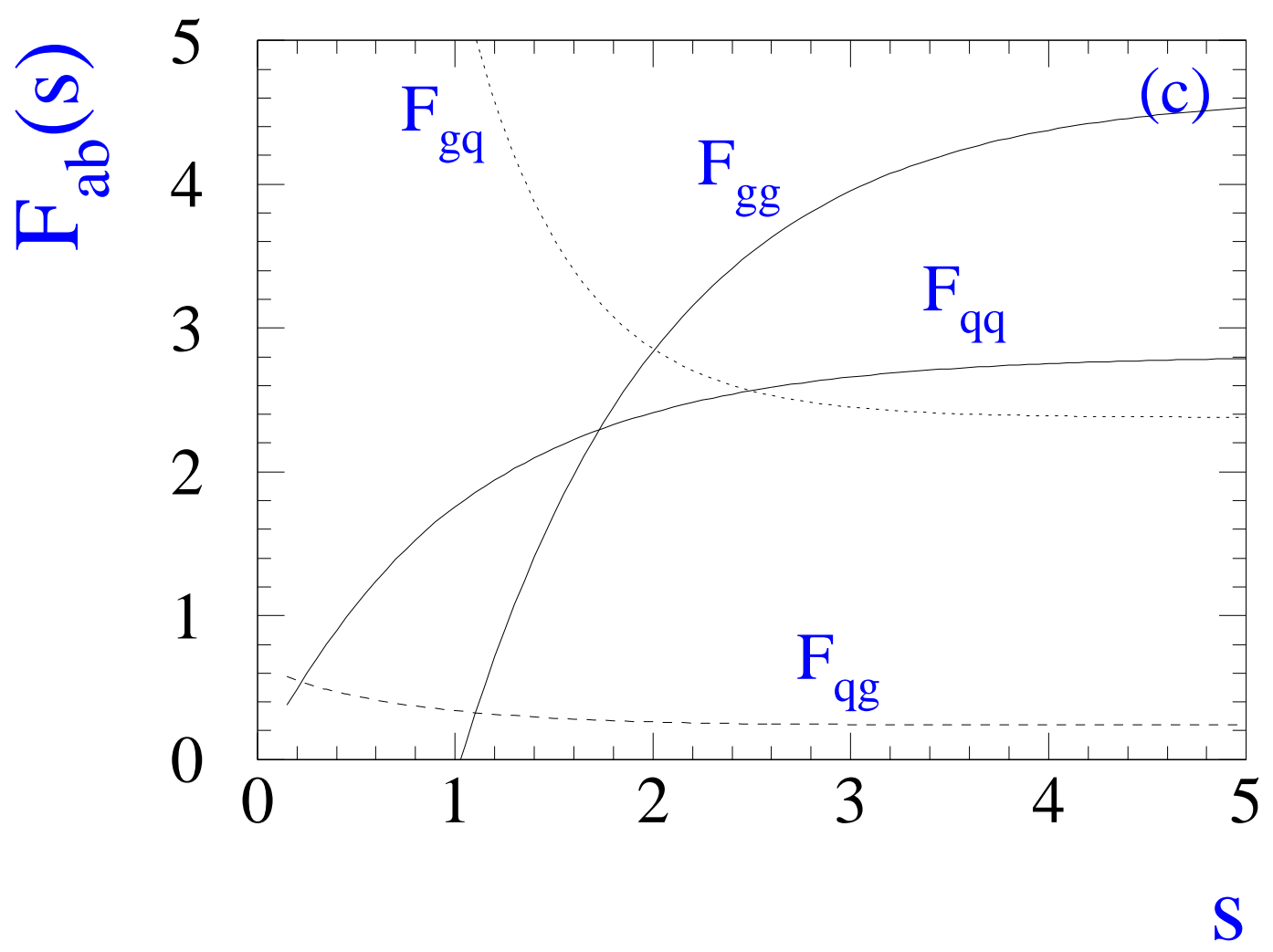


This figure "fig1-2.png" is available in "png" format from: http://arxiv.org/ps/hep-ph/9403409v1 
This figure "fig2-2.png" is available in "png" format from: http://arxiv.org/ps/hep-ph/9403409v1 
This figure "fig3-2.png" is available in "png" format from: http://arxiv.org/ps/hep-ph/9403409v1 


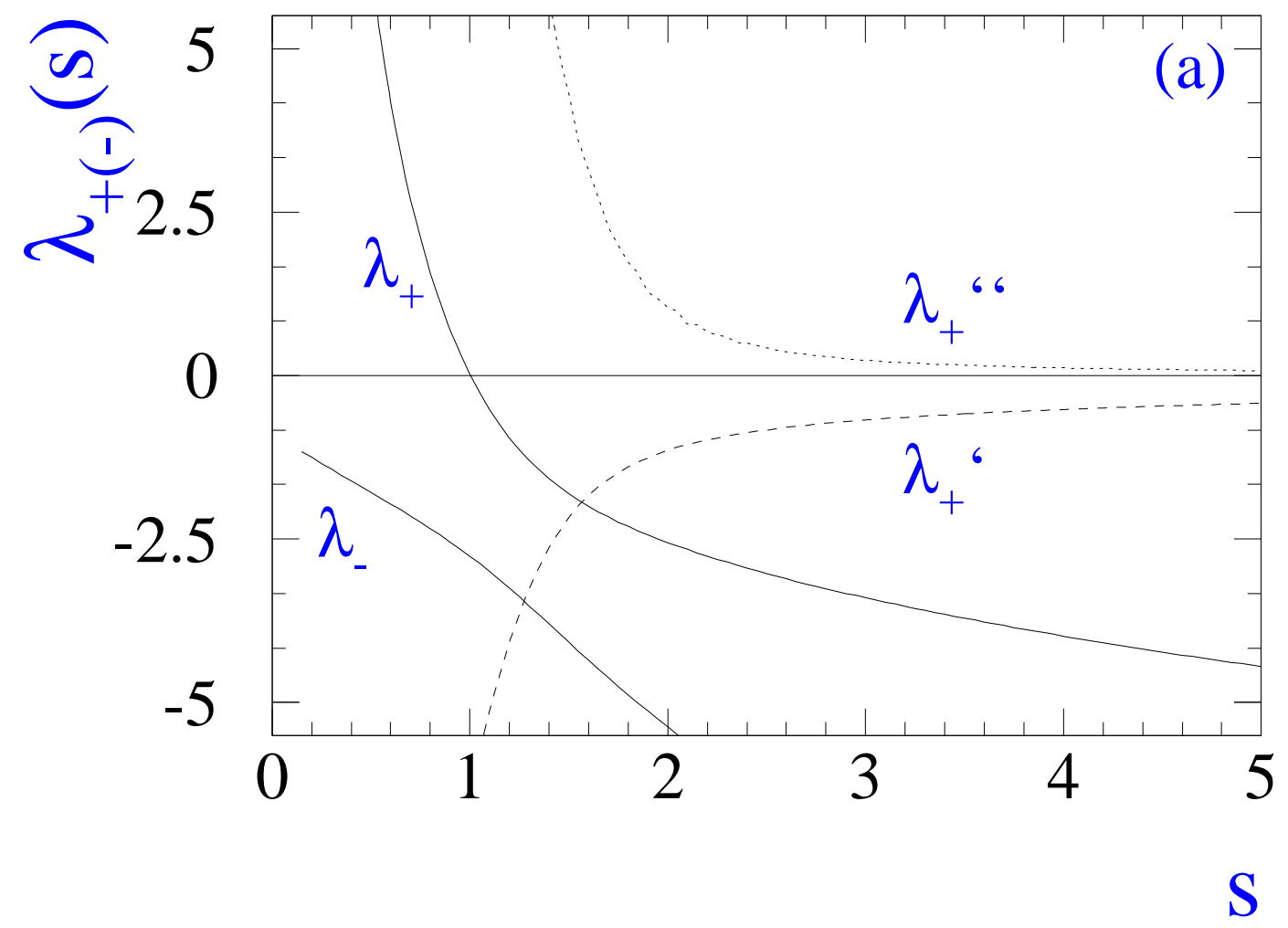




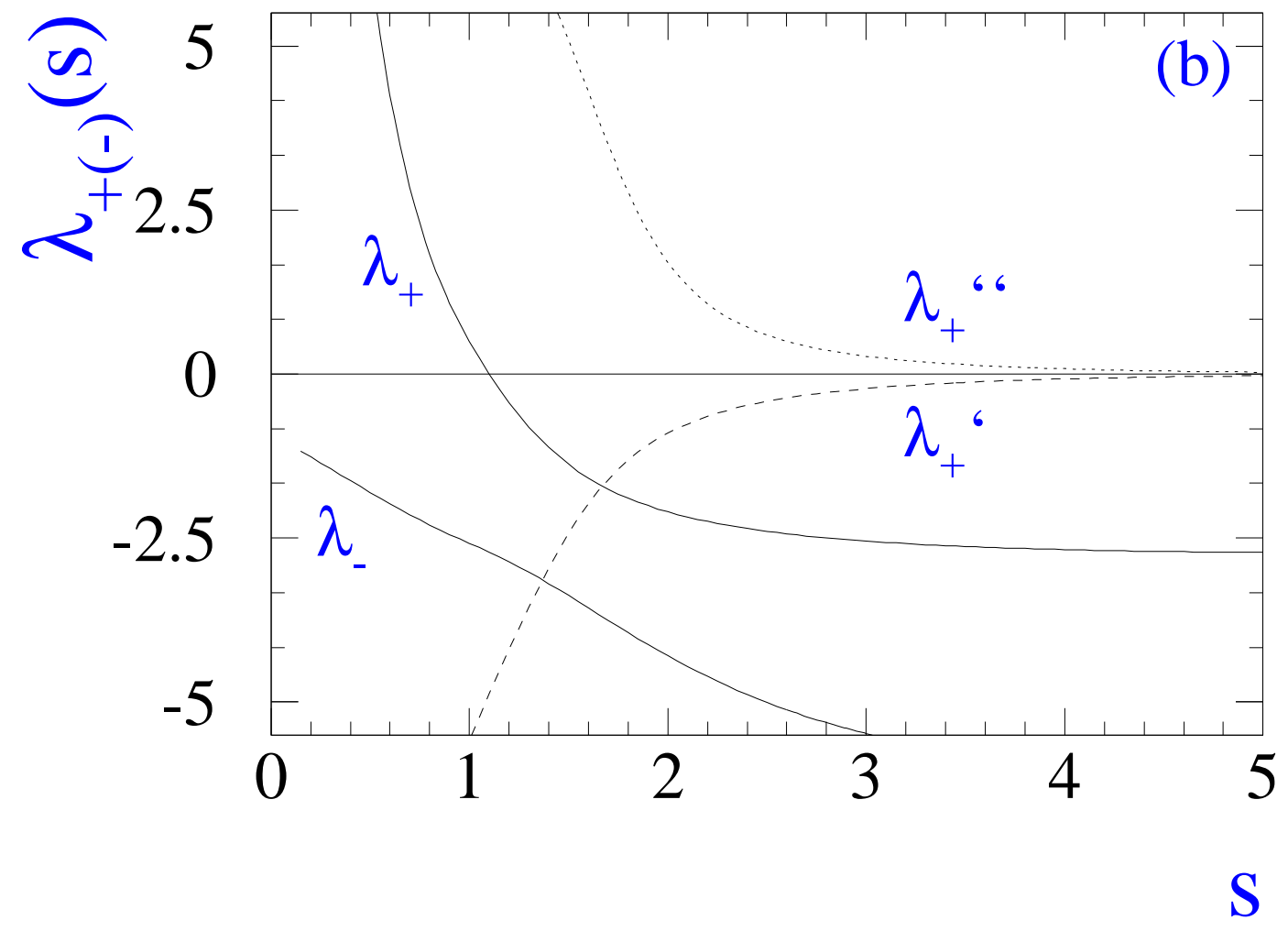




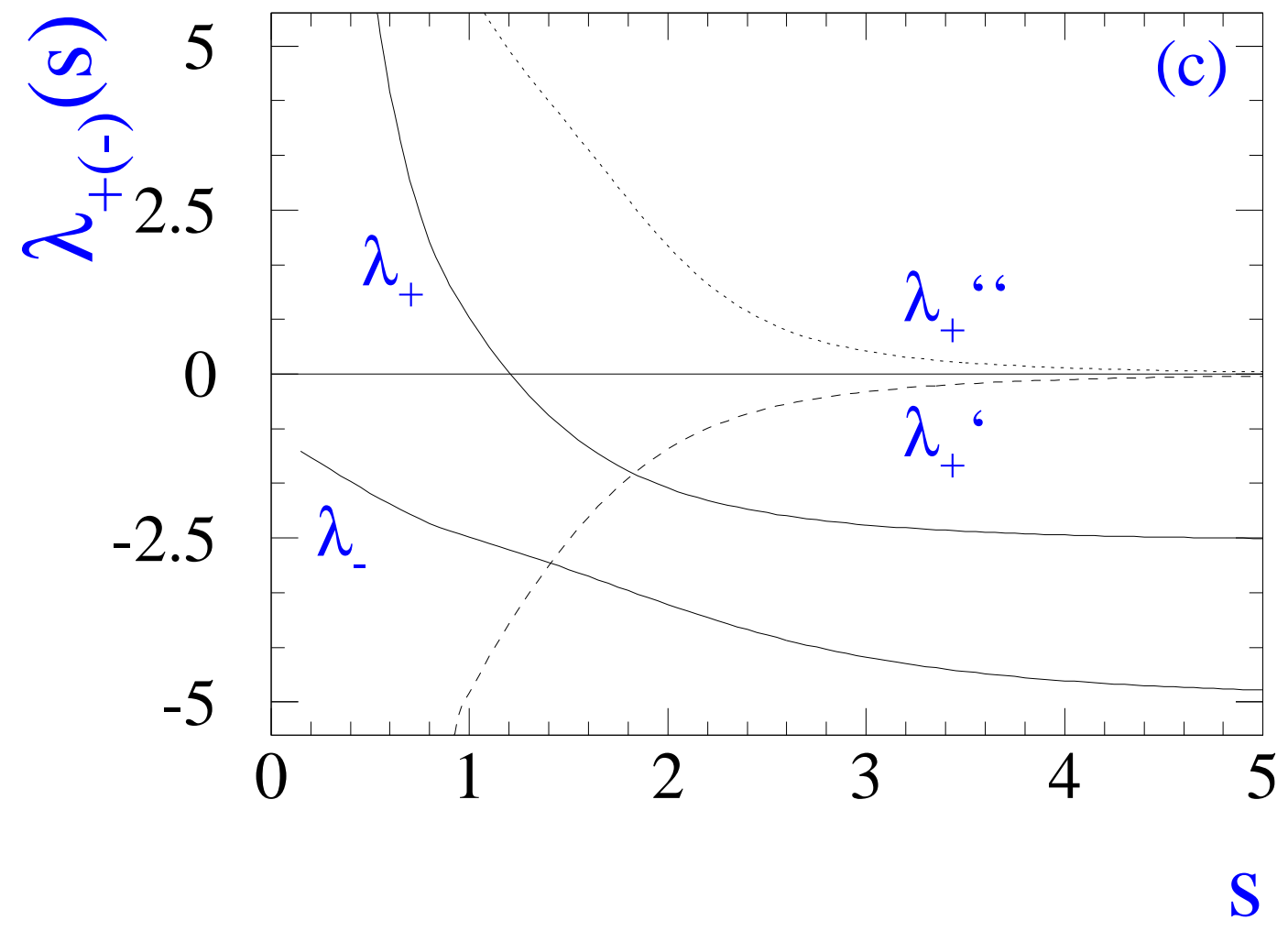


This figure "fig1-3.png" is available in "png" format from: http://arxiv.org/ps/hep-ph/9403409v1 
This figure "fig2-3.png" is available in "png" format from: http://arxiv.org/ps/hep-ph/9403409v1 
This figure "fig3-3.png" is available in "png" format from: http://arxiv.org/ps/hep-ph/9403409v1 


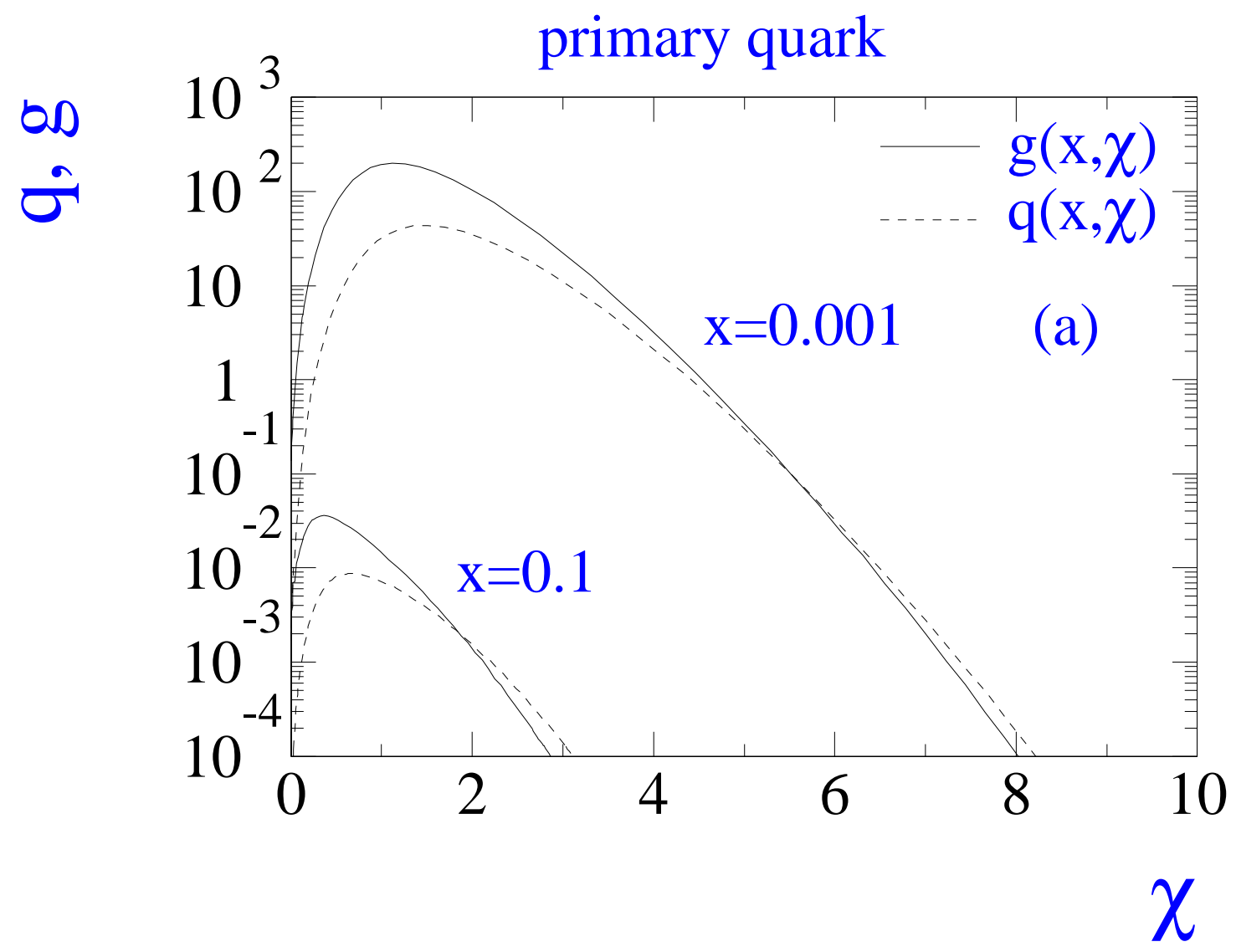




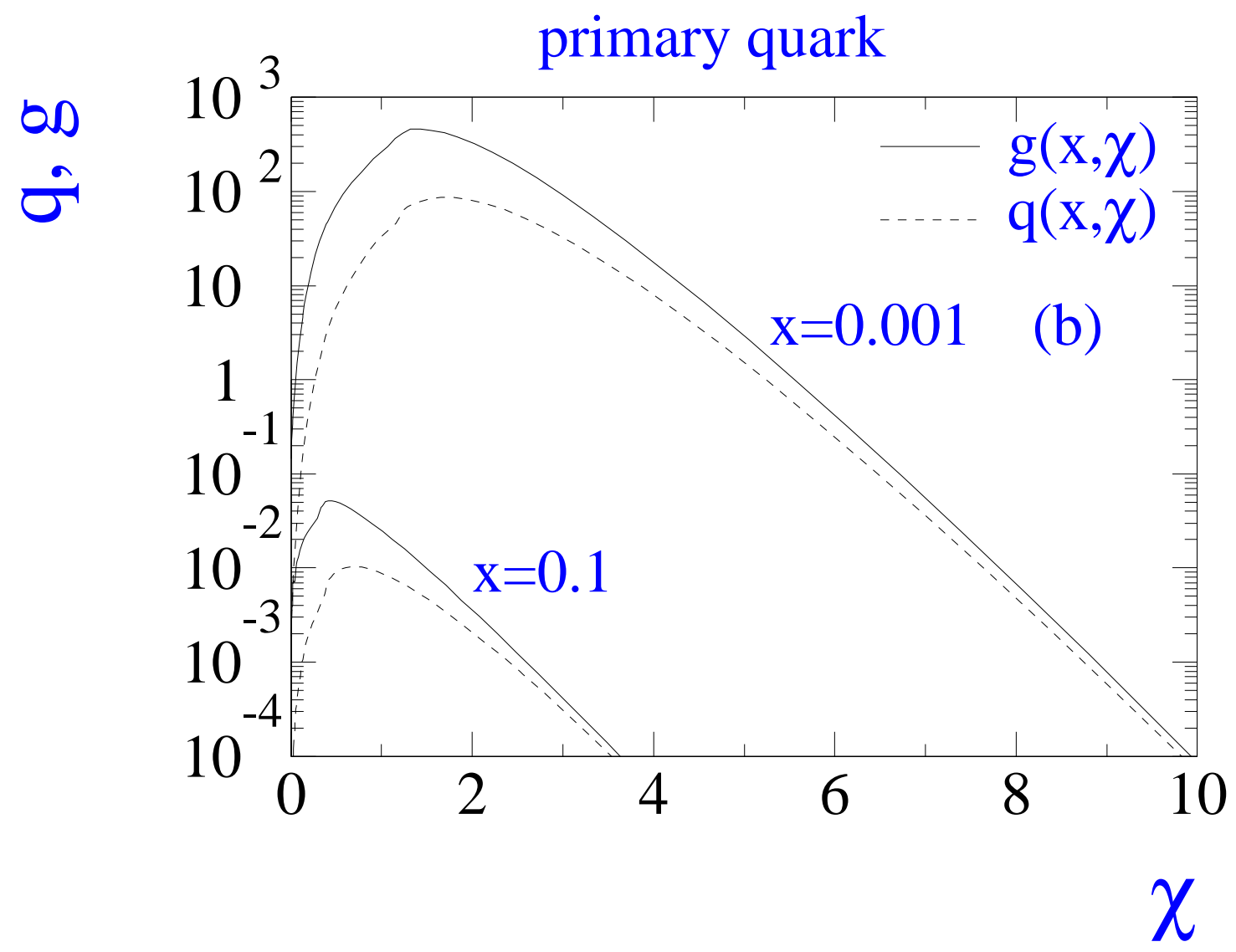




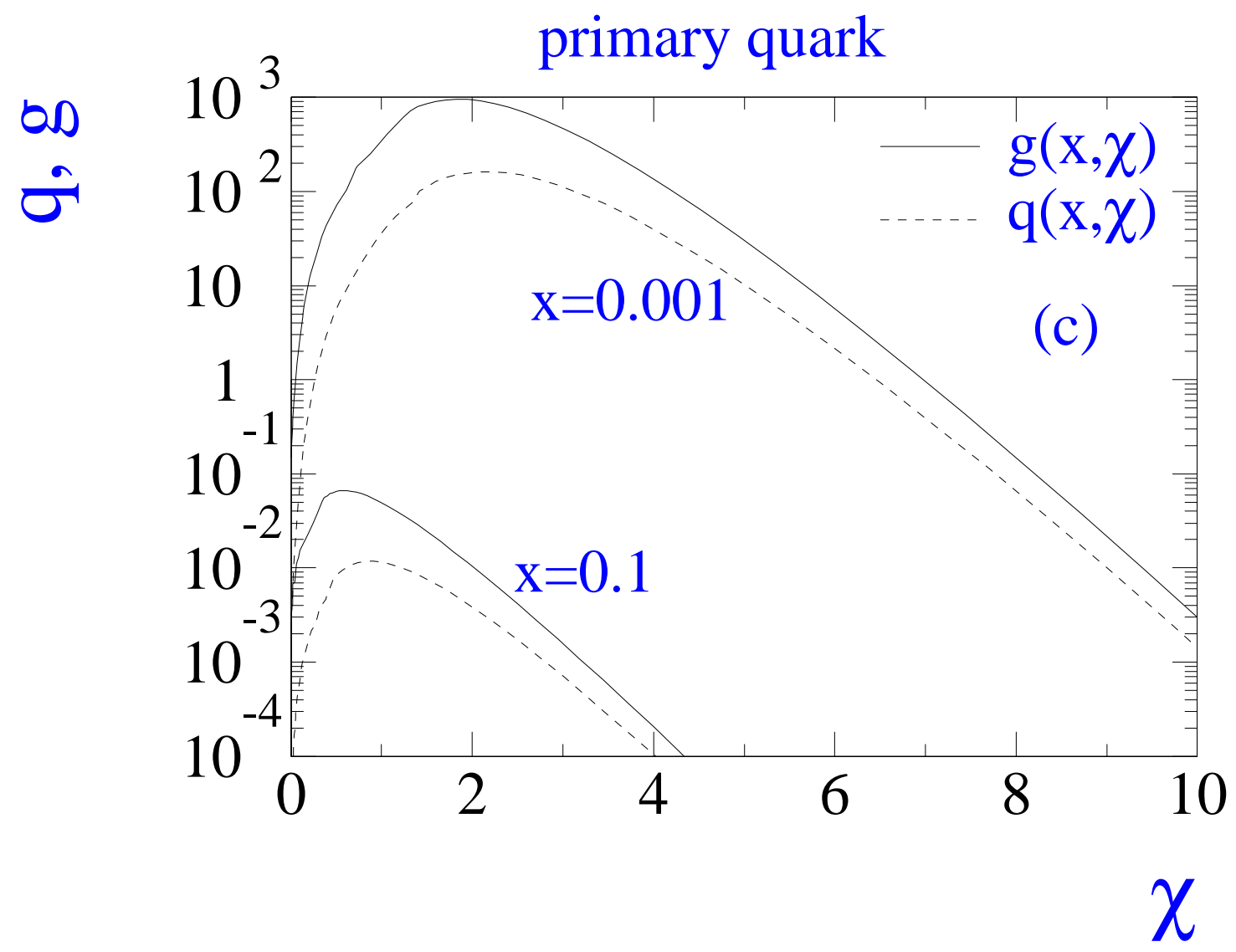


This figure "fig1-4.png" is available in "png" format from: http://arxiv.org/ps/hep-ph/9403409v1 
This figure "fig2-4.png" is available in "png" format from: http://arxiv.org/ps/hep-ph/9403409v1 
This figure "fig3-4.png" is available in "png" format from: http://arxiv.org/ps/hep-ph/9403409v1 


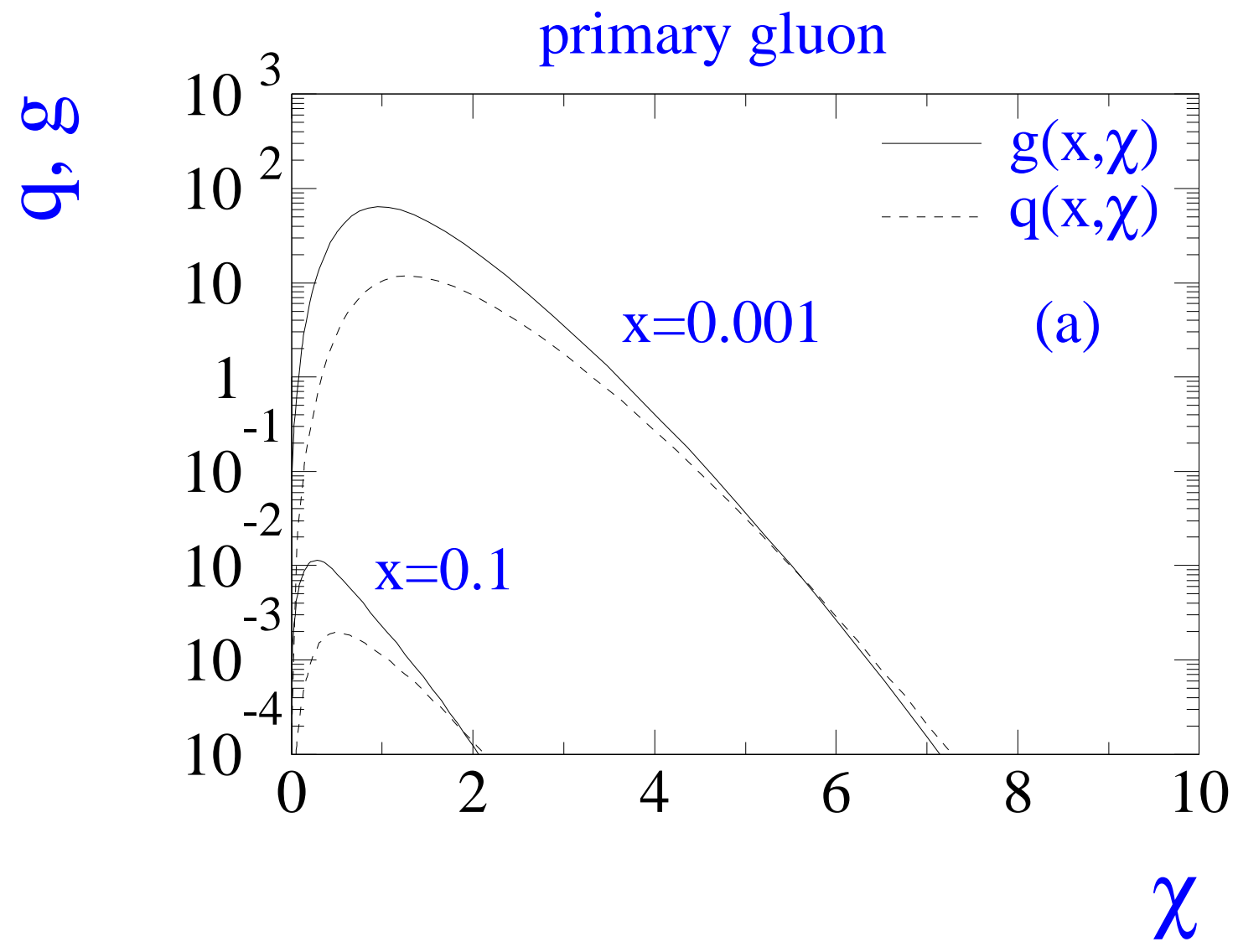




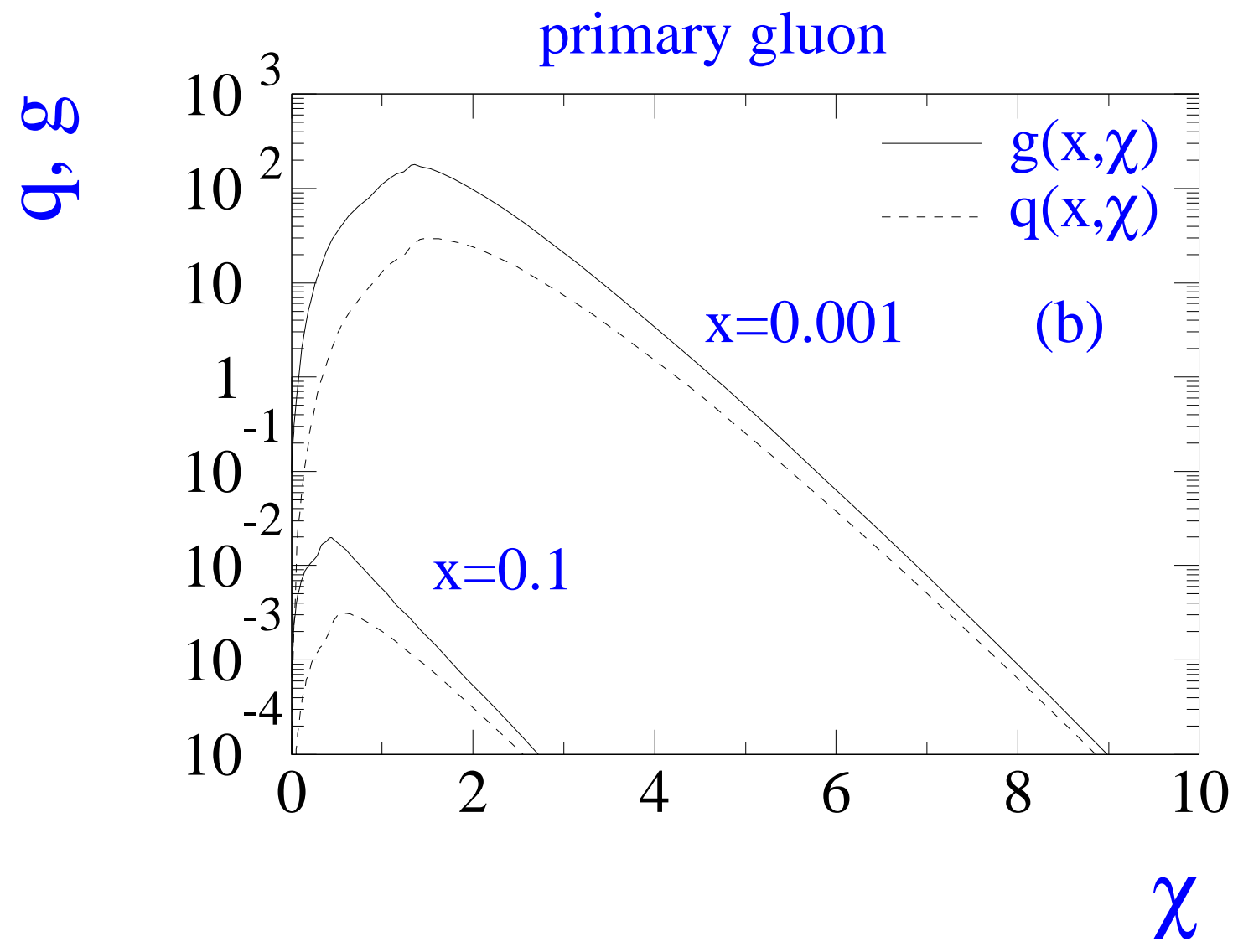




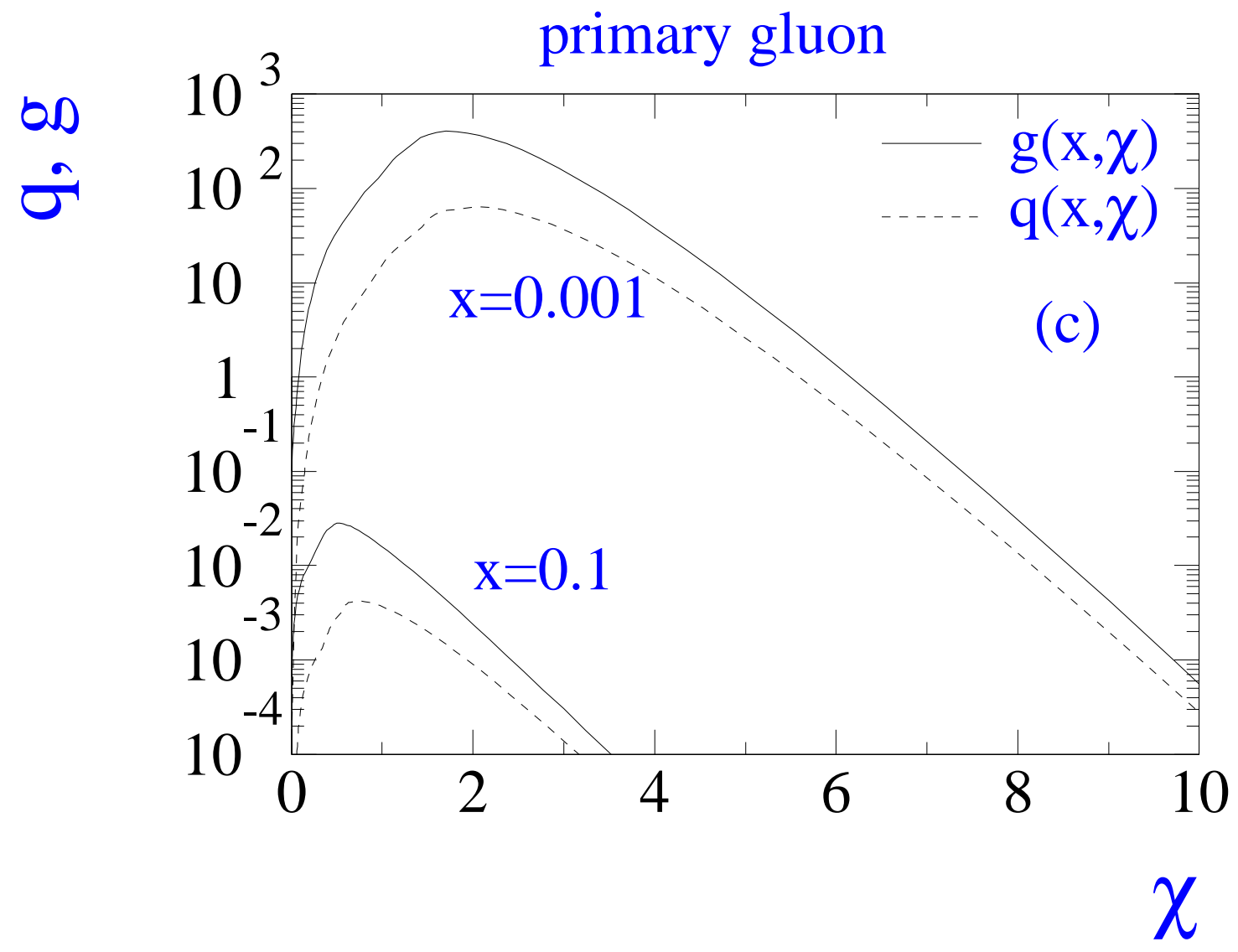


This figure "fig1-5.png" is available in "png" format from: http://arxiv.org/ps/hep-ph/9403409v1 
This figure "fig2-5.png" is available in "png" format from: http://arxiv.org/ps/hep-ph/9403409v1 
This figure "fig3-5.png" is available in "png" format from: http://arxiv.org/ps/hep-ph/9403409v1 


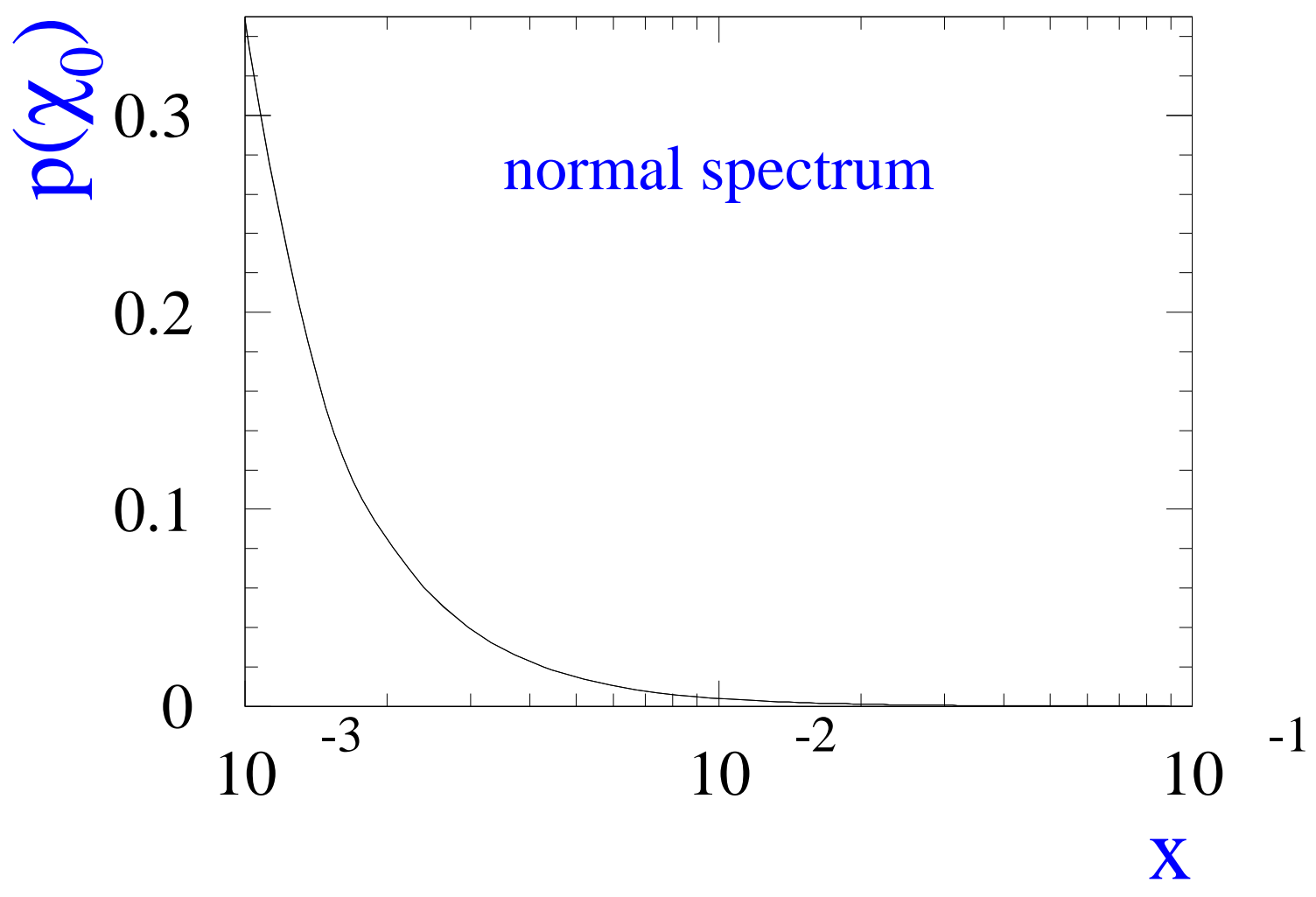




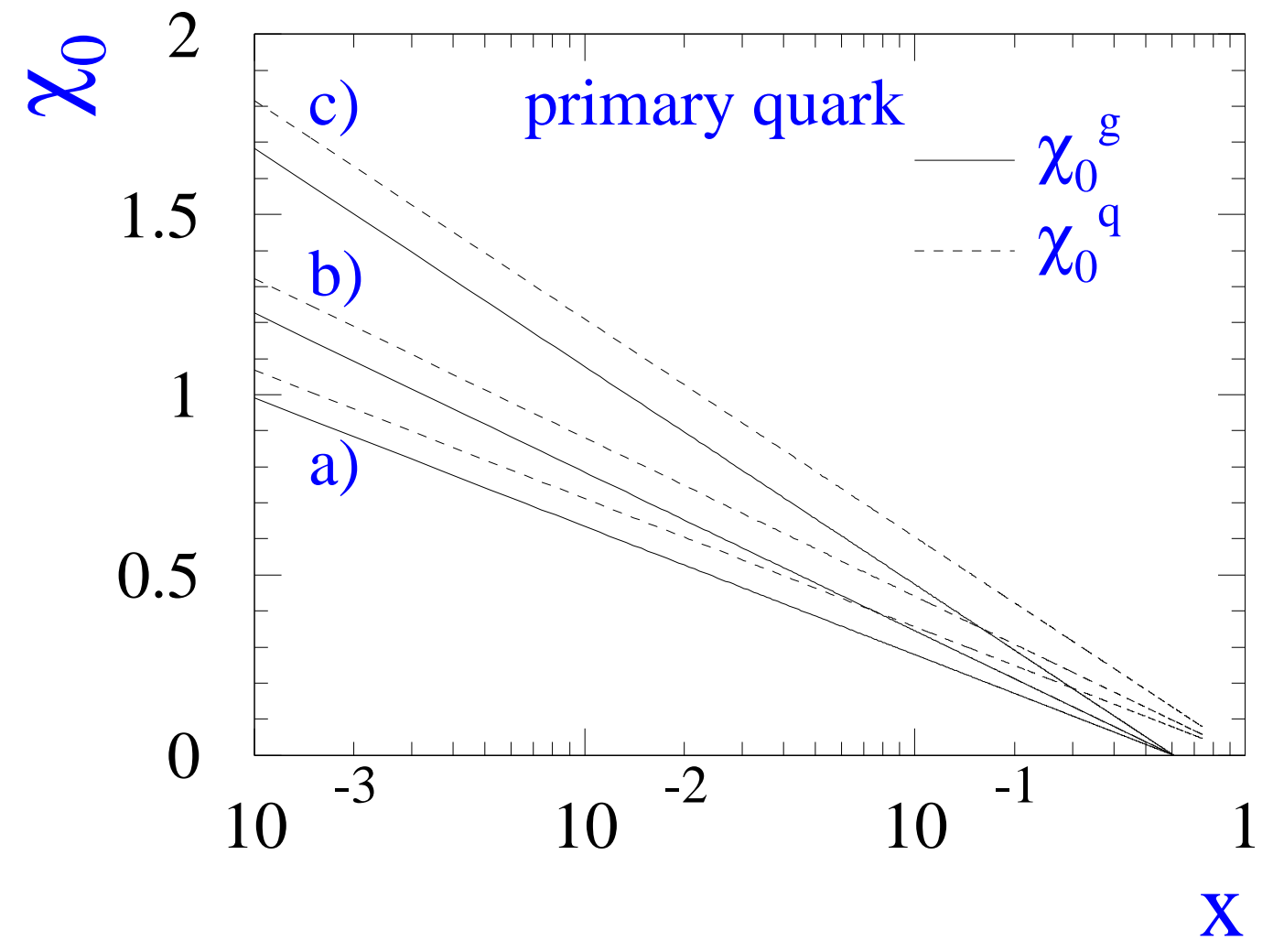




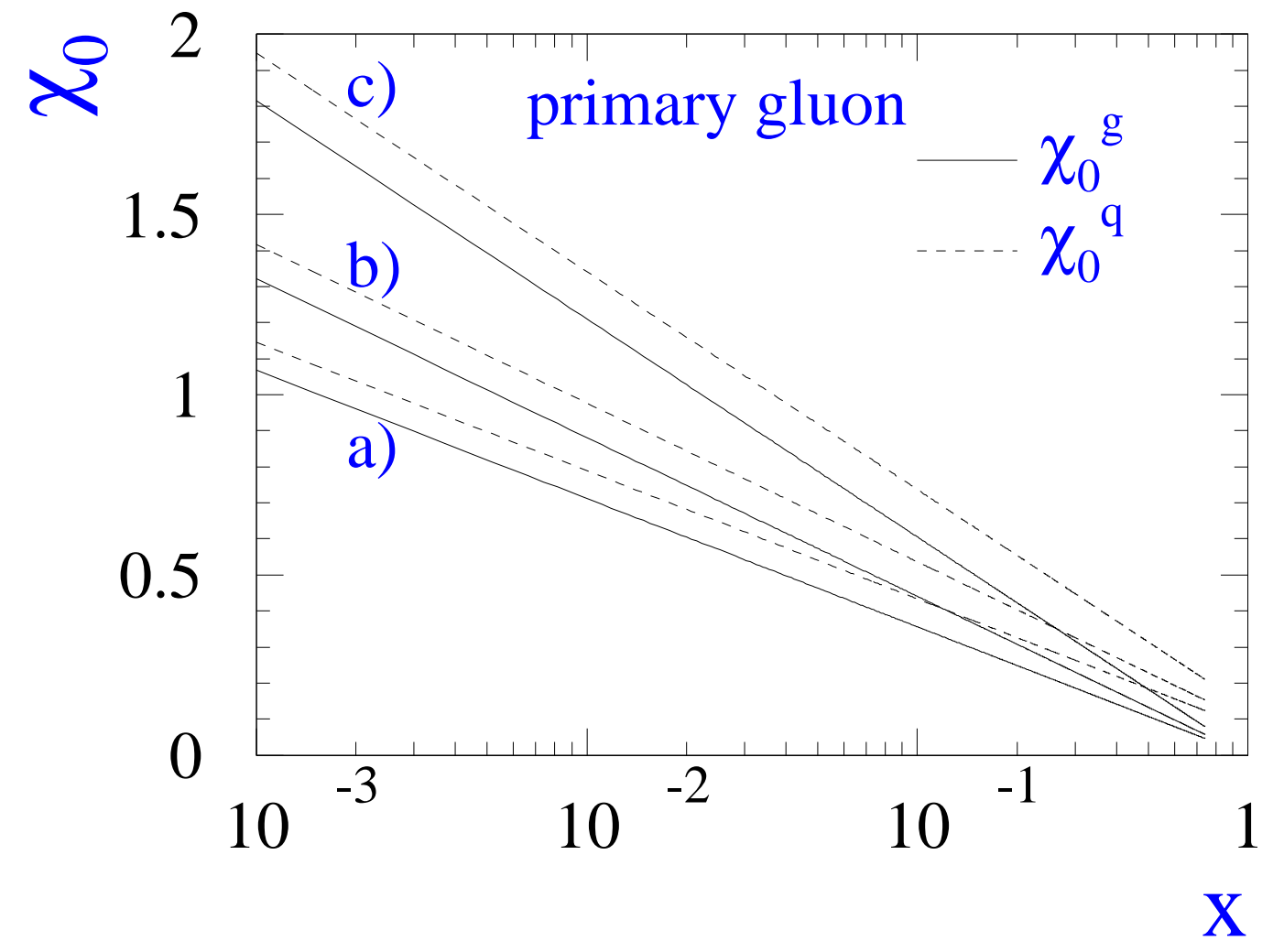


This figure "fig3-6.png" is available in "png" format from: http://arxiv.org/ps/hep-ph/9403409v1 